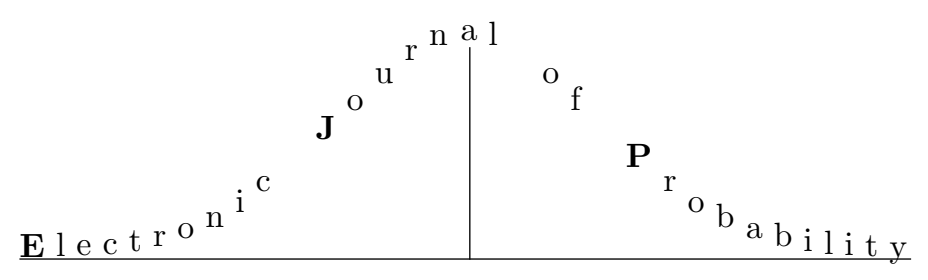

Vol. 11 (2006), Paper no. 35, pages 893-933.

Journal URL

http://www.math. washington.edu/ ejpecp/

\title{
Post-gelation behavior of a spatial coagulation model
}

\author{
Wolfgang Wagner \\ Weierstrass Institute for \\ Applied Analysis and Stochastics \\ Mohrenstrasse 39 \\ D-10117 Berlin, Germany \\ wagner@wias-berlin.de
}

\begin{abstract}
A coagulation model on a finite spatial grid is considered. Particles of discrete masses jump randomly between sites and, while located at the same site, stick together according to some coagulation kernel. The asymptotic behavior (for increasing particle numbers) of this model is studied in the situation when the coagulation kernel grows sufficiently fast so that the phenomenon of gelation is observed. Weak accumulation points of an appropriate sequence of measure-valued processes are characterized in terms of solutions of a nonlinear equation. A natural description of the behavior of the gel is obtained by using the onepoint compactification of the size space. Two aspects of the limiting equation are of special interest. First, for a certain class of coagulation kernels, this equation differs from a naive extension of Smoluchowski's coagulation equation. Second, due to spatial inhomogeneity, an equation for the time evolution of the gel mass density has to be added. The jump rates are assumed to vanish with increasing particle masses so that the gel is immobile. Two different gel growth mechanisms (active and passive gel) are found depending on the type of the coagulation kernel.
\end{abstract}

Key words: Spatial coagulation model; post-gelation behavior; stochastic particle systems

AMS 2000 Subject Classification: Primary 60K40.

Submitted to EJP on June 2 2006, final version accepted September 182006. 


\section{Introduction}

We consider a particle system

$$
\left(x_{i}^{N}(t), \alpha_{i}^{N}(t)\right), \quad i=1, \ldots, n^{N}(t), \quad t \geq 0 .
$$

The state space of a single particle is

$$
\mathcal{Z}=\{1,2, \ldots\} \times G
$$

where $G$ is a finite set of (spatial) locations. Particles jump between sites

$$
(x, \alpha) \rightarrow(x, \beta)
$$

according to some rate function and, while located at the same site, stick together

$$
(x, \alpha),(y, \alpha) \rightarrow(x+y, \alpha)
$$

following stochastic rules determined by some coagulation kernel. The index $N=1,2, \ldots$. denotes the number of monomers (units of size 1) in the system so that

$$
\sum_{i=1}^{n^{N}(t)} x_{i}^{N}(t)=N, \quad \forall t \geq 0 .
$$

The discrete (both in space and size) model described above was used in [27] as an approximation to the spatially continuous coagulation equation with diffusion

$$
\begin{aligned}
& \frac{\partial}{\partial t} c(t, k, r)=D(k) \Delta_{r} c(t, k, r)+ \\
& \quad \frac{1}{2} \sum_{x+y=k} K(x, y) c(t, x, r) c(t, y, r)-c(t, k, r) \sum_{y=1}^{\infty} K(k, y) c(t, y, r) .
\end{aligned}
$$

The solution $c(t, k, r)$ is interpreted as the average number density of clusters of size $k$ at time $t$ and position $r$. The symbol $\Delta_{r}$ denotes the Laplace operator with respect to the position variable, $D(k)$ are size-dependent diffusion coefficients and $K$ is the coagulation kernel. If there is no dependence on $r$ (spatial homogeneity), then the diffusion term disappears and equation (1.4) reduces to Smoluchowski's coagulation equation 30]

$$
\begin{aligned}
& \frac{d}{d t} c(t, k)= \\
& \quad \frac{1}{2} \sum_{x+y=k} K(x, y) c(t, x) c(t, y)-c(t, k) \sum_{y=1}^{\infty} K(k, y) c(t, y),
\end{aligned}
$$

when considering the specific kernel

$$
K(x, y)=\left(x^{-1 / 3}+y^{-1 / 3}\right)\left(x^{1 / 3}+y^{1 / 3}\right) .
$$


Theoretical investigations of the gelation phenomenon go back to the paper [10] on condensation polymerization. Flory studied the size distribution of polymers and established critical conditions (in terms of a parameter called "extent of reaction") for the formation of "infinitely large" molecules (gel). Developing this approach, Stockmayer [26] pointed out a connection of the polymer size distribution with equation (1.5), where

$$
K(x, y)=[(f-2) x+2][(f-2) y+2] .
$$

Polymeric molecules ( $k$-mers) are composed of $k$ monomeric units. Each monomeric unit carries $f$ functional groups capable of reacting with each other. Thus, the kernel 1.7 represents the number of possible links between $x$-mers and $y$-mers. Note that an equation with the commonly used multiplicative kernel

$$
K(x, y)=x y
$$

can be obtained from equation (1.5) with the kernel (1.7) in the limit $f \rightarrow \infty$, when time is appropriately scaled. Stockmayer [26] argued with Flory about the correct post-gelation behavior and proposed a solution different from Flory's. Early reviews of the subject were given in [11] and [12, Ch. IX]. An extended discussion of different solutions after the gel point and corresponding modified equations can be found in [32] $(f=3)$ and [34] $(f>2)$. The paper [32] contains a rather complete list of relevant earlier references.

Rigorous results concerning the derivation of the spatially inhomogeneous coagulation equation (1.4) from systems of diffusing spherical particles, interacting at contact, were obtained in [18] (constant kernel) and 24] (kernel (1.6)). Stochastic models of coagulation in the spatially homogeneous case go back to [22], [14, [21]. In those papers the coagulation kernel, which contains the information about the microscopic behavior of the physical system, is postulated. An extended review of the subject was given in [2]. We also refer to the recent paper [13] studying the spatially homogeneous case with rather general gelling kernels. When combining the Marcus-Lushnikov approach with spatial inhomogeneity, particles coagulate with a certain rate when they are close enough to each other (e.g., in the same cell). Convergence results for such models with non-gelling kernels were obtained in [15] (bounded kernel) and [3] (sub-linear kernel). The two-site case of the van Dongen model described above (with $D(k)=1$ and kernel (1.8)) was studied in [25]. Analytical results concerning the coagulation equation with diffusion (1.4) (and references to earlier studies) can be found, e.g., in [19] and [20] (see also [8, Section 8]). Equation (1.4) with constant diffusion coefficients $D(k)=1$ and the multiplicative kernel (1.8) was studied in [17]. The following equation for the gel mass density $g(t, r)$ was suggested,

$$
\frac{\partial}{\partial t} g(t, r)=\Delta_{r} g(t, r)+R
$$

where

$$
R=\lim _{k \rightarrow \infty} \sum_{x=1}^{k} \sum_{y=k-x+1}^{\infty} x^{2} c(t, x, r) y c(t, y, r)
$$

is a "Radon measure describing the rate of gel production". Considering diffusion coefficients $D(k)$ vanishing sufficiently fast (with $k \rightarrow \infty$ ) and the multiplicative kernel (1.8), van Dongen 
[27] proposed a modification of equation (1.4), namely

$$
\begin{aligned}
& \frac{\partial}{\partial t} c(t, k, r)=D(k) \Delta_{r} c(t, k, r)+ \\
& \quad \frac{1}{2} \sum_{x+y=k} x y c(t, x, r) c(t, y, r)-k c(t, k, r)\left[\sum_{y=1}^{\infty} y c(t, y, r)+g(t, r)\right]
\end{aligned}
$$

where the time evolution of the gel mass density is determined by the equation

$$
\frac{\partial}{\partial t} g(t, r)=g(t, r) \sum_{k=1}^{\infty} k^{2} c(t, k, r) \text {. }
$$

The paper is organized as follows. In Section 2 the asymptotic behavior (as $N \rightarrow \infty$ ) of the particle system (1.1) is studied. Weak accumulation points of an appropriately scaled sequence of measure-valued processes (based on (1.1)) are shown to be concentrated on the set of solutions of a nonlinear equation. The results cover the situation, when the coagulation kernel grows sufficiently fast so that the phenomenon of gelation is observed. Using the one-point compactification of the size space and considering mass density instead of number density leads to a natural description of the behavior of the gel under rather general assumptions on the coagulation kernel. Section 3 is concerned with properties of the limiting equation. Two aspects are of special interest. First, for a certain class of coagulation kernels, this equation differs from a naive extension of Smoluchowski's coagulation equation (as, e.g., (1.11) compared to (1.4)). Second, an equation for the time evolution of the gel mass density has to be added (as, e.g., (1.9) or (1.12). Note that the second aspect is absent in the spatially homogeneous situation, since the gel mass density is determined just as the mass defect of the solution $c(t, k)$. In the spatially inhomogeneous situation the gel is distributed over different sites and gel equations are of interest. In general, they describe both the spatial motion and the growth of the gel. In this paper the case of vanishing diffusion coefficients is considered so that the gel is immobile. The growth behavior depends on the kernel and is determined by terms of the type occurring in 1.10 or 1.12 . Finally, Section 4 contains most of the technical proofs.

\section{Asymptotic behavior of the stochastic model}

We represent the particle system (1.1) in form of measures

$$
X^{N}(t, d x, d \alpha)=\frac{1}{N} \sum_{i=1}^{n^{N}(t)} x_{i}^{N}(t) \delta_{\left(x_{i}^{N}(t), \alpha_{i}^{N}(t)\right)}(d x, d \alpha)
$$

on the state space $(1.2)$, where $\delta_{z}$ denotes the delta-measure concentrated in $z \in \mathcal{Z}$. The transition kernel of the corresponding jump process is

$$
\begin{aligned}
\lambda^{N}(\mu, B)= & \sum_{i=1}^{n} \sum_{\beta \in G} \kappa\left(x_{i}, \alpha_{i}, \beta\right) 1_{B}\left(J_{1}(\mu, i, \beta)\right)+ \\
& \frac{1}{2 N} \sum_{1 \leq i \neq j \leq n} \delta_{\alpha_{i}, \alpha_{j}} K\left(x_{i}, x_{j}, \alpha_{i}\right) 1_{B}\left(J_{2}(\mu, i, j)\right)
\end{aligned}
$$


where $1_{B}$ denotes the indicator function of a set $B, \delta_{\alpha, \beta}$ is Kronecker's symbol, $\kappa$ and $K$ are non-negative functions on $\{1,2, \ldots\} \times G^{2}$ and $\{1,2, \ldots\}^{2} \times G$, respectively, and

$$
\begin{aligned}
J_{1}(\mu, i, \beta) & =\mu+\frac{1}{N}\left[x_{i} \delta_{\left(x_{i}, \beta\right)}-x_{i} \delta_{\left(x_{i}, \alpha_{i}\right)}\right] \\
J_{2}(\mu, i, j) & =\mu+\frac{1}{N}\left[\left(x_{i}+x_{j}\right) \delta_{\left(x_{i}+x_{j}, \alpha_{i}\right)}-x_{i} \delta_{\left(x_{i}, \alpha_{i}\right)}-x_{j} \delta_{\left(x_{j}, \alpha_{j}\right)}\right]
\end{aligned}
$$

are jump transformations. The kernel $(2.2)$ is defined on the state space of the process (2.1) (cf. $(1.2)$,

$$
\begin{aligned}
E^{N} & = \\
& \left\{\frac{1}{N} \sum_{i=1}^{n} x_{i} \delta_{\left(x_{i}, \alpha_{i}\right)}: n \geq 1,\left(x_{i}, \alpha_{i}\right) \in \mathcal{Z}, i=1, \ldots, n, \sum_{i=1}^{n} x_{i}=N\right\} .
\end{aligned}
$$

It satisfies

$$
\begin{aligned}
\lambda^{N}\left(\mu, E^{N}\right) & =\sum_{i=1}^{n} \sum_{\beta \in G} \kappa\left(x_{i}, \alpha_{i}, \beta\right)+\frac{1}{2 N} \sum_{1 \leq i \neq j \leq n} \delta_{\alpha_{i}, \alpha_{j}} K\left(x_{i}, x_{j}, \alpha_{i}\right) \\
& \leq N\left[\sup _{1 \leq x \leq N, \alpha \in G} \sum_{\beta \in G} \kappa(x, \alpha, \beta)+\sup _{1 \leq x, y \leq N, \alpha \in G} K(x, y, \alpha)\right]
\end{aligned}
$$

The pathwise behavior of the process in terms of particles is obtained from the kernel (2.2). The jump process is regular, since the kernel is bounded.

Consider the space

$$
\mathcal{Z}^{\prime}=(\{1,2, \ldots\} \cup\{\infty\}) \times G,
$$

where $\{1,2, \ldots\} \cup\{\infty\}$ is the one-point compactification of $\{1,2, \ldots\}$. Continuous functions $\varphi \in \mathcal{C}\left(\mathcal{Z}^{\prime}\right)$ are functions on $\mathcal{Z}$ (cf. $(1.2)$ ) with finite limits

$$
\varphi(\infty, \alpha):=\lim _{x \rightarrow \infty} \varphi(x, \alpha), \quad \forall \alpha \in G .
$$

Let $\mathcal{P}\left(\mathcal{Z}^{\prime}\right)$ denote the space of probability measures on $\mathcal{Z}^{\prime}$ equipped with the topology of weak convergence. For $\varphi \in \mathcal{C}\left(\mathcal{Z}^{\prime}\right)$ and $\mu \in \mathcal{P}\left(\mathcal{Z}^{\prime}\right)$, we introduce the notations

$$
\langle\varphi, \mu\rangle=\int_{\mathcal{Z}^{\prime}} \varphi(x, \alpha) \mu(d x, d \alpha)
$$

and

$$
\begin{aligned}
\mathcal{G}(\varphi, \mu)= & \int_{\mathcal{Z}^{\prime}} \sum_{\beta \in G} \kappa(x, \alpha, \beta)[\varphi(x, \beta)-\varphi(x, \alpha)] \mu(d x, d \alpha)+ \\
& \frac{1}{2} \int_{\mathcal{Z}^{\prime}} \int_{\mathcal{Z}^{\prime}} \delta_{\alpha, \beta} F_{\varphi}(x, y, \alpha) \mu(d x, d \alpha) \mu(d y, d \beta),
\end{aligned}
$$

where

$$
\begin{aligned}
& F_{\varphi}(x, y, \alpha)= \\
& \quad \frac{K(x, y, \alpha)}{x y}[(x+y) \varphi(x+y, \alpha)-x \varphi(x, \alpha)-y \varphi(y, \alpha)], \quad x, y<\infty,
\end{aligned}
$$


and

$$
\begin{aligned}
F_{\varphi}(\infty, y, \alpha) & =[\varphi(\infty, \alpha)-\varphi(y, \alpha)] \lim _{x \rightarrow \infty} \frac{K(x, y, \alpha)}{x}, \\
F_{\varphi}(x, \infty, \alpha) & =F_{\varphi}(\infty, x, \alpha), \quad F_{\varphi}(\infty, \infty, \alpha)=0, \\
\kappa(\infty, \alpha, \beta) & =\lim _{x \rightarrow \infty} \kappa(x, \alpha, \beta) .
\end{aligned}
$$

Theorem 2.1. Assume

$$
\lim _{x \rightarrow \infty} \kappa(x, \alpha, \beta)=0, \quad \forall \alpha, \beta \in G .
$$

Let $K$ be symmetric and such that

$$
\lim _{x \rightarrow \infty} \frac{K(x, y, \alpha)}{x}<\infty, \quad \forall \alpha \in G, \quad y=1,2, \ldots
$$

and

$$
\max _{\alpha \in G} K(x, y, \alpha) \leq C_{K} x y, \quad \forall x, y=1,2, \ldots,
$$

for some $C_{K}>0$. Assume

$$
X^{N}(0) \Rightarrow \nu_{0} \quad \text { for some } \quad \nu_{0} \in \mathcal{P}\left(\mathcal{Z}^{\prime}\right),
$$

where the sign $\Rightarrow$ denotes convergence in distribution.

Then the processes (2.1) form a relatively compact sequence of random variables with values in $\mathcal{D}\left([0, \infty), \mathcal{P}\left(\mathcal{Z}^{\prime}\right)\right)$, where $\mathcal{D}$ denotes the Skorokhod space of right-continuous functions with left limits. Every weak accumulation point $X$ solves, almost surely, the limiting equation

$$
\langle\varphi, X(t)\rangle=\left\langle\varphi, \nu_{0}\right\rangle+\int_{0}^{t} \mathcal{G}(\varphi, X(s)) d s, \quad \forall t \geq 0
$$

for all $\varphi$ such that (cf. Remark 2.2)

$$
\varphi(x, \alpha)=c_{0}(\varphi, \alpha), \quad \forall x \geq \bar{x}(\varphi), \quad \alpha \in G .
$$

Moreover, $X$ is almost surely continuous.

Remark 2.2. Condition (2.15) means that the test functions are constant for sufficiently large arguments, which is stronger than just continuity (cf. 2.6)).

Remark 2.3. Continuity of $\mathcal{G}(\varphi, \mu)$ with respect to $\mu$ follows from the continuity of the functions $\kappa$ and $F_{\varphi}$. The function $\kappa$ is continuous, according to (2.10). The function (2.8), (2.9) is continuous if 2.11) holds and $\varphi$ has the form 2.15).

Remark 2.4. Note that (2.12) does not follow from (2.11). Indeed, consider the kernel $K(x, y)=x^{3}$, if $x=y$, and 1 , otherwise. 
Remark 2.5. The measure-valued process (2.1) represents the particle mass concentration. It counts the number of monomers (mass) of particles of a given size instead of the number of particles of a given size (particle number concentration) considered, e.g., in [5]. Note that the underlying particle process (1.1) has the "direct simulation" dynamics, not the "mass flow" dynamics considered, e.g., in [4] and [31, Sect. 3]. The process [2.1] turned out to be most appropriate for studying the post-gelation behavior.

Remark 2.6. Theorem 2.1 implies existence of solutions of equation (2.14). Very little is known about uniqueness of post-gelation solutions. However, in Section 3 properties are obtained for any solution of the limiting equation. One particular uniqueness result will be mentioned in Section 3.3.1.

\section{Properties of the limiting equation}

Let

$$
\nu \in \mathcal{C}\left([0, \infty), \mathcal{P}\left(\mathcal{Z}^{\prime}\right)\right)
$$

be any solution of equation (2.14) (cf. Remark 2.6 and (2.5)).

\subsection{Derivation of strong equations}

Expression (2.7) takes the form

$$
\begin{aligned}
\mathcal{G}(\varphi, \mu)= & \sum_{x=1}^{\infty} \sum_{\alpha, \beta \in G} \kappa(x, \alpha, \beta)[\varphi(x, \beta)-\varphi(x, \alpha)] \mu(x, \alpha)+ \\
& \sum_{\alpha, \beta \in G} \kappa(\infty, \alpha, \beta)[\varphi(\infty, \beta)-\varphi(\infty, \alpha)] \mu(\infty, \alpha)+ \\
& \frac{1}{2} \sum_{x, y=1}^{\infty} \sum_{\alpha \in G} F_{\varphi}(x, y, \alpha) \mu(x, \alpha) \mu(y, \alpha)+ \\
& \frac{1}{2} \sum_{\alpha \in G} F_{\varphi}(\infty, \infty, \alpha) \mu(\infty, \alpha) \mu(\infty, \alpha)+ \\
& \frac{1}{2} \sum_{y=1}^{\infty} \sum_{\alpha \in G} F_{\varphi}(\infty, y, \alpha) \mu(\infty, \alpha) \mu(y, \alpha)+ \\
& \frac{1}{2} \sum_{x=1}^{\infty} \sum_{\alpha \in G} F_{\varphi}(x, \infty, \alpha) \mu(\infty, \alpha) \mu(x, \alpha)
\end{aligned}
$$


Taking into account symmetry of $K$ (and therefore $\left.F_{\varphi}\right),(2.9)$ and $(2.10)$, one obtains

$$
\begin{gathered}
\mathcal{G}(\varphi, \mu)=\sum_{x=1}^{\infty} \sum_{\alpha \neq \beta} \kappa(x, \alpha, \beta)[\varphi(x, \beta)-\varphi(x, \alpha)] \mu(x, \alpha)+ \\
\frac{1}{2} \sum_{x, y=1}^{\infty} \sum_{\alpha \in G} \frac{K(x, y, \alpha)}{x y}[(x+y) \varphi(x+y, \alpha)-x \varphi(x, \alpha)-y \varphi(y, \alpha)] \times \\
\quad \mu(x, \alpha) \mu(y, \alpha)+ \\
\sum_{y=1}^{\infty} \sum_{\alpha \in G}[\varphi(\infty, \alpha)-\varphi(y, \alpha)] \tilde{K}(\infty, y, \alpha) \mu(\infty, \alpha) \mu(y, \alpha),
\end{gathered}
$$

where (cf. 2.11)

$$
\tilde{K}(\infty, y, \alpha)=\lim _{x \rightarrow \infty} \frac{K(x, y, \alpha)}{x} .
$$

Remark 3.1. The solution (3.1) satisfies equation (2.14) for any test function $\varphi$ of the form

$$
d_{k, \gamma}(x, \alpha)=\delta_{k, x} \delta_{\gamma, \alpha}, \quad d_{k, \gamma}(\infty, \alpha)=0
$$

and

$$
\psi_{k, \gamma}(x, \alpha)=1_{[k, \infty)}(x) \delta_{\gamma, \alpha}, \quad \psi_{k, \gamma}(\infty, \alpha)=\delta_{\gamma, \alpha},
$$

where $k=1,2, \ldots$ and $\gamma \in G$, since these functions satisfy 2.15).

\subsubsection{Sol equations}

One obtains from 3.2 , with $\varphi$ of the form (3.4), that

$$
\begin{aligned}
& \mathcal{G}\left(d_{k, \gamma}, \mu\right)=\sum_{\alpha \neq \gamma} \kappa(k, \alpha, \gamma) \mu(k, \alpha)-\sum_{\beta \neq \gamma} \kappa(k, \gamma, \beta) \mu(k, \gamma)+ \\
& \frac{1}{2} \sum_{x+y=k} \frac{K(x, y, \gamma)}{x y}(x+y) \mu(x, \gamma) \mu(y, \gamma)- \\
& \frac{1}{2} \sum_{y=1}^{\infty} \frac{K(k, y, \gamma)}{k y} k \mu(k, \gamma) \mu(y, \gamma)- \\
& \frac{1}{2} \sum_{x=1}^{\infty} \frac{K(x, k, \gamma)}{x k} k \mu(x, \gamma) \mu(k, \gamma)-\tilde{K}(\infty, k, \gamma) \mu(\infty, \gamma) \mu(k, \gamma) \\
&= \sum_{\alpha \neq \gamma} \kappa(k, \alpha, \gamma) \mu(k, \alpha)-\mu(k, \gamma) \sum_{\beta \neq \gamma} \kappa(k, \gamma, \beta)+ \\
& \frac{k}{2} \sum_{x+y=k} \frac{K(x, y, \gamma)}{x y} \mu(x, \gamma) \mu(y, \gamma)- \\
& \mu(k, \gamma)\left[\sum_{x=1}^{\infty} \frac{K(x, k, \gamma)}{x} \mu(x, \gamma)+\tilde{K}(\infty, k, \gamma) \mu(\infty, \gamma)\right] .
\end{aligned}
$$


Equation (2.14), with the representation (3.6), provides the sol equations

$$
\begin{aligned}
& \nu(t, k, \gamma)=\nu(0, k, \gamma)+\int_{0}^{t}\left[\sum_{\alpha \neq \gamma} \kappa(k, \alpha, \gamma) \nu(s, k, \alpha)-\right. \\
& \nu(s, k, \gamma) \sum_{\beta \neq \gamma} \kappa(k, \gamma, \beta)+\frac{k}{2} \sum_{x=1}^{k-1} \frac{K(x, k-x, \gamma)}{x(k-x)} \nu(s, x, \gamma) \nu(s, k-x, \gamma)- \\
& \left.\nu(s, k, \gamma) \sum_{x=1}^{\infty} \frac{K(x, k, \gamma)}{x} \nu(s, x, \gamma)-\nu(s, k, \gamma) \tilde{K}(\infty, k, \gamma) \nu(s, \infty, \gamma)\right] d s, \\
& \forall t \geq 0, \quad k=1,2, \ldots, \quad \gamma \in G .
\end{aligned}
$$

\subsubsection{Gel equations}

Now we are going to obtain equations for $\nu(t, \infty, \gamma), \gamma \in G$. Note that

$$
\nu(t, \infty, \gamma)=\lim _{k \rightarrow \infty}\left\langle\psi_{k, \gamma}, \nu(t)\right\rangle, \quad \forall t \geq 0,
$$

where the functions $\psi_{k, \gamma}$ are defined in (3.5). The starting point is the system of equations (cf. Remark 3.1)

$$
\begin{aligned}
\left\langle\psi_{k, \gamma}, \nu(t)\right\rangle= & \left\langle\psi_{k, \gamma}, \nu(0)\right\rangle+\int_{0}^{t} \mathcal{G}\left(\psi_{k, \gamma}, \nu(s)\right) d s \\
& \forall t \geq 0, \quad k=1,2, \ldots
\end{aligned}
$$

It follows from $(3.8)$ and $(3.9)$ that

$$
\exists \lim _{k \rightarrow \infty} \int_{0}^{t} \mathcal{G}\left(\psi_{k, \gamma}, \nu(s)\right) d s .
$$


One obtains from $(3.2)$, with $\varphi$ of the form 3.5 , that

$$
\begin{aligned}
& \mathcal{G}\left(\psi_{k, \gamma}, \mu\right)=\sum_{x=k}^{\infty}\left[\sum_{\alpha \neq \gamma} \kappa(x, \alpha, \gamma) \mu(x, \alpha)-\mu(x, \gamma) \sum_{\beta \neq \gamma} \kappa(x, \gamma, \beta)\right]+ \\
& \frac{1}{2} \sum_{x=1}^{k-1} \sum_{y=k-x}^{k-1} \frac{K(x, y, \gamma)}{x y}(x+y) \mu(x, \gamma) \mu(y, \gamma)+ \\
& \frac{1}{2} \sum_{x=1}^{k-1} \sum_{y=k}^{\infty} \frac{K(x, y, \gamma)}{x y} x \mu(x, \gamma) \mu(y, \gamma)+ \\
& \frac{1}{2} \sum_{y=1}^{k-1} \sum_{x=k}^{\infty} \frac{K(x, y, \gamma)}{x y} y \mu(x, \gamma) \mu(y, \gamma)+\mu(\infty, \gamma) \sum_{y=1}^{k-1} \tilde{K}(\infty, y, \gamma) \mu(y, \gamma) \\
& =\sum_{x=k}^{\infty}\left[\sum_{\alpha \neq \gamma} \kappa(x, \alpha, \gamma) \mu(x, \alpha)-\mu(x, \gamma) \sum_{\beta \neq \gamma} \kappa(x, \gamma, \beta)\right]+ \\
& \sum_{x=1}^{k-1} \sum_{y=k-x}^{k-1} \frac{K(x, y, \gamma)}{y} \mu(x, \gamma) \mu(y, \gamma)+ \\
& \sum_{x=1}^{k-1} \sum_{y=k}^{\infty} \frac{K(x, y, \gamma)}{y} \mu(x, \gamma) \mu(y, \gamma)+\mu(\infty, \gamma) \sum_{y=1}^{k-1} \tilde{K}(\infty, y, \gamma) \mu(y, \gamma) \\
& =\sum_{x=k}^{\infty}\left[\sum_{\alpha \neq \gamma} \kappa(x, \alpha, \gamma) \mu(x, \alpha)-\mu(x, \gamma) \sum_{\beta \neq \gamma} \kappa(x, \gamma, \beta)\right]+ \\
& \sum_{x=1}^{k-1} \mu(x, \gamma) \sum_{y=k-x}^{\infty} \frac{K(x, y, \gamma)}{y} \mu(y, \gamma)+\mu(\infty, \gamma) \sum_{y=1}^{k-1} \tilde{K}(\infty, y, \gamma) \mu(y, \gamma)
\end{aligned}
$$

Since

$$
S_{1}(k, \gamma, \mu):=\sum_{x=k}^{\infty}\left[\sum_{\alpha \neq \gamma} \kappa(x, \alpha, \gamma) \mu(x, \alpha)-\mu(x, \gamma) \sum_{\beta \neq \gamma} \kappa(x, \gamma, \beta)\right]
$$

satisfies (cf. (2.10)

$$
\left|S_{1}(k, \gamma, \mu)\right| \leq 2 \max _{x \geq 1} \sum_{\alpha, \beta \in G} \kappa(x, \alpha, \beta)<\infty,
$$

the dominated convergence theorem implies

$$
\lim _{k \rightarrow \infty} \int_{0}^{t} S_{1}(k, \gamma, \nu(s)) d s=\int_{0}^{t} \lim _{k \rightarrow \infty} S_{1}(k, \gamma, \nu(s)) d s=0 .
$$

Since

$$
S_{2}(k, \gamma, \mu):=\mu(\infty, \gamma) \sum_{y=1}^{k-1} \tilde{K}(\infty, y, \gamma) \mu(y, \gamma)
$$


is non-decreasing in $k$ and

$$
S(k, \gamma, \mu):=\sum_{x=1}^{k-1} \mu(x, \gamma) \sum_{y=k-x}^{\infty} \frac{K(x, y, \gamma)}{y} \mu(y, \gamma)
$$

is non-negative, it follows from $(3.10)-(3.12)$ that

$$
\begin{aligned}
& \exists \lim _{k \rightarrow \infty} \int_{0}^{t} S_{2}(k, \gamma, \nu(s)) d s= \\
& \quad \int_{0}^{t} \nu(s, \infty, \gamma) \sum_{y=1}^{\infty} \tilde{K}(\infty, y, \gamma) \nu(s, y, \gamma) d s<\infty
\end{aligned}
$$

Finally, it follows from $3.10-3.12$ and $(3.14)$ that

$$
\exists \lim _{k \rightarrow \infty} \int_{0}^{t} S(k, \gamma, \nu(s)) d s<\infty .
$$

Thus, one obtains from (3.8), 3.9) and 3.11) 3.15 the gel equations

$$
\begin{aligned}
\nu(t, \infty, \gamma)= & \nu(0, \infty, \gamma)+\int_{0}^{t} \nu(s, \infty, \gamma) \sum_{y=1}^{\infty} \tilde{K}(\infty, y, \gamma) \nu(s, y, \gamma) d s+ \\
& \lim _{k \rightarrow \infty} \int_{0}^{t}\left[\sum_{x=1}^{k-1} \nu(s, x, \gamma) \sum_{y=k-x}^{\infty} \frac{K(x, y, \gamma)}{y} \nu(s, y, \gamma)\right] d s \\
& \forall t \geq 0, \quad \gamma \in G .
\end{aligned}
$$

Remark 3.2. The case $\nu(0, \infty, \gamma)>0$ (for some $\gamma \in G$ ) is covered by Theorem 2.1.

\subsection{Properties of the gel solution}

Taking into account (3.14) and (3.15), one concludes from (3.16) that

$$
\begin{aligned}
\nu(t, \infty, \gamma)= & \nu(u, \infty, \gamma)+\int_{u}^{t} \nu(s, \infty, \gamma) \sum_{y=1}^{\infty} \tilde{K}(\infty, y, \gamma) \nu(s, y, \gamma) d s+ \\
& \lim _{k \rightarrow \infty} \int_{u}^{t} S(k, \gamma, \nu(s)) d s, \quad \forall 0 \leq u \leq t .
\end{aligned}
$$

According to (3.17), the growth of the gel may originate from two different sources. In the case $\tilde{K}=0$ (cf. 3.3$)$ ), the gel is "passive" and grows due to the "gel production term"

$$
\begin{aligned}
& \lim _{k \rightarrow \infty} \int_{u}^{t} S(k, \gamma, \nu(s)) d s= \\
& \quad \lim _{k \rightarrow \infty} \int_{u}^{t}\left[\sum_{x=1}^{k-1} \nu(s, x, \gamma) \sum_{y=k-x}^{\infty} \frac{K(x, y, \gamma)}{y} \nu(s, y, \gamma)\right] d s
\end{aligned}
$$


which depends only on the sol solution. In the case $\tilde{K}>0$, the gel "actively" collects mass from the sol solution, according to the term

$$
\int_{u}^{t} \nu(s, \infty, \gamma) \sum_{y=1}^{\infty} \tilde{K}(\infty, y, \gamma) \nu(s, y, \gamma) d s .
$$

It turns out that the gel production term (3.18) vanishes in the active gel case (under some additional assumptions).

\subsubsection{Estimates of the gel production term}

Here we study the behavior of the term (3.18). The proofs of the lemmas will be given in Section 4 .

First we find sufficient conditions assuring a vanishing gel production term.

Lemma 3.3. Let $0 \leq u<t$ and $\gamma \in G$. Assume the kernel satisfies

$$
K(x, y, \gamma) \leq C x y, \quad \forall x, y=1,2, \ldots,
$$

for some $C>0$. If

$$
\sum_{y=1}^{\infty} y \nu(u, y, \gamma)<\infty
$$

then

$$
\lim _{k \rightarrow \infty} S(k, \gamma, \nu(u))=0
$$

Moreover, if the sol solution satisfies

$$
\int_{u}^{t}\left[\sum_{y=1}^{\infty} y \nu(s, y, \gamma)\right] d s<\infty
$$

then

$$
\lim _{k \rightarrow \infty} \int_{u}^{t} S(k, \gamma, \nu(s)) d s=0 .
$$

Lemma 3.4. Let $0 \leq u<t$ and $\gamma \in G$. Assume the kernel satisfies

$$
K(x, y, \gamma) \leq C\left[x y^{a}+x^{a} y\right], \quad \forall x, y=1,2, \ldots,
$$

for some $C>0$ and $a \in[0,1]$. If

$$
\sum_{y=1}^{\infty} y^{a} \nu(u, y, \gamma)<\infty \quad \text { and } \quad \lim _{y \rightarrow \infty} y \nu(u, y, \gamma)=0
$$


then (3.19) holds. Moreover, if the sol solution satisfies

$$
\int_{u}^{t}\left[\sum_{y=1}^{\infty} y^{a} \nu(s, y, \gamma)\right] d s<\infty
$$

and

$$
\lim _{y \rightarrow \infty}\left[y \sup _{s \in[u, t]} \nu(s, y, \gamma)\right]=0
$$

then (3.21) holds.

Lemma 3.5. Let $0 \leq u<t$ and $\gamma \in G$. Assume the kernel satisfies

$$
K(x, y, \gamma) \leq C\left[x^{a} y^{b}+x^{b} y^{a}\right], \quad \forall x, y=1,2, \ldots,
$$

for some $C>0$ and $a, b \in[0,1]$.

(i) Assume

$$
\nu(u, y, \gamma) \leq \tilde{C} y^{\beta}, \quad \forall y=1,2, \ldots,
$$

for some $\tilde{C}>0$ and $\beta<-1$. Then

$$
\sup _{k} S(k, \gamma, \nu(u))<\infty \quad \text { if } \quad \beta \leq-\frac{a+b+1}{2}
$$

and

$$
\lim _{k \rightarrow \infty} S(k, \gamma, \nu(u))=0 \quad \text { if } \quad \beta<-\frac{a+b+1}{2} .
$$

(ii) Assume the sol solution satisfies

$$
\nu(s, y, \gamma) \leq \tilde{C}(s) y^{\beta}, \quad \forall s \in[u, t], \quad y=1,2, \ldots,
$$

for some $\beta<-1$, where

$$
\int_{u}^{t} \tilde{C}(s)^{2} d s<\infty
$$

Then

$$
\int_{u}^{t} \sup _{k} S(k, \gamma, \nu(s)) d s<\infty \quad \text { if } \quad \beta \leq-\frac{a+b+1}{2}
$$

and

$$
\int_{u}^{t} \limsup _{k \rightarrow \infty} S(k, \gamma, \nu(s)) d s=0 \quad \text { if } \quad \beta<-\frac{a+b+1}{2} .
$$


Remark 3.6. Note that (3.24) implies (3.21). If $a=b=1$ and $\beta<-2$, then Lemma 3.5 follows from Lemma 3.3. If $a=1$ and $\beta<-1-b$, then Lemma 3.5 follows from Lemma 3.4.

Finally, we provide conditions assuring a non-vanishing gel production term.

Lemma 3.7. Let $0 \leq u<t$ and $\gamma \in G$. Assume the kernel satisfies

$$
K(x, y, \gamma) \geq C\left[x^{a} y^{b}+x^{b} y^{a}\right], \quad \forall x, y=1,2, \ldots,
$$

for some $C>0$ and $a, b \in[0,1]: a+b>1$.

(i) Assume

$$
\nu(u, y, \gamma) \geq \tilde{C} y^{\beta}, \quad \forall y=1,2, \ldots,
$$

for some $\tilde{C}>0$ and $\beta<-1$. Then

$$
\liminf _{k \rightarrow \infty} S(k, \gamma, \nu(u))>0 \quad \text { if } \quad \beta=-\frac{a+b+1}{2},
$$

and

$$
\lim _{k \rightarrow \infty} S(k, \gamma, \nu(u))=\infty \quad \text { if } \quad-\frac{a+b+1}{2}<\beta<-1 .
$$

(ii) Assume the sol solution satisfies

$$
\nu(s, y, \gamma) \geq \tilde{C}(s) y^{\beta}, \quad \forall s \in[u, t], \quad y=1,2, \ldots,
$$

for some $\beta<-1$, where

$$
\int_{u}^{t} \tilde{C}(s)^{2} d s>0
$$

Then

$$
\int_{u}^{t} \liminf _{k \rightarrow \infty} S(k, \gamma, \nu(s)) d s>0 \quad \text { if } \quad \beta=-\frac{a+b+1}{2},
$$

and

$$
\int_{u}^{t} \liminf _{k \rightarrow \infty} S(k, \gamma, \nu(s)) d s=\infty \quad \text { if } \quad-\frac{a+b+1}{2}<\beta<-1 .
$$

Remark 3.8. Since

$$
\int_{0}^{t} \liminf _{k \rightarrow \infty} S(k, \gamma, \nu(s)) d s \leq \liminf _{k \rightarrow \infty} \int_{0}^{t} S(k, \gamma, \nu(s)) d s
$$

according to Fatou's lemma, (3.27) and (3.28) imply

$$
\lim _{k \rightarrow \infty} \int_{u}^{t} S(k, \gamma, \nu(s)) d s>0
$$

and

$$
\lim _{k \rightarrow \infty} \int_{u}^{t} S(k, \gamma, \nu(s)) d s=\infty,
$$

respectively. Note that (3.30) contradicts (3.15) so that (3.25), 3.26) can not be fulfilled if

$$
-\frac{a+b+1}{2}<\beta<-1 \text {. }
$$




\subsubsection{Active gel case}

Here we provide sufficient conditions assuring that the gel solution satisfies the equation

$$
\begin{aligned}
& \nu(t, \infty, \gamma)=\nu(\tau(\gamma), \infty, \gamma)+ \\
& \int_{\tau(\gamma)}^{t} \nu(s, \infty, \gamma) \sum_{y=1}^{\infty} \tilde{K}(\infty, y, \gamma) \nu(s, y, \gamma) d s, \quad \forall t \geq \tau(\gamma)
\end{aligned}
$$

where

$$
\tau(\gamma):=\inf \{t>0: \nu(t, \infty, \gamma)>0\}, \quad \gamma \in G .
$$

Note that $\nu(t, \infty, \gamma)$ is non-decreasing in $t$, according to (3.17). Moreover, the gel solution satisfies

$$
\nu(s, \infty, \gamma)=0, \quad \forall s<\tau(\gamma), \quad \nu(s, \infty, \gamma)>0, \quad \forall s>\tau(\gamma) .
$$

Theorem 3.9. Let $\gamma \in G$. Assume the kernel satisfies

$$
K(x, y, \gamma) \leq C x y, \quad \forall x, y=1,2, \ldots,
$$

and (cf. 3.3)

$$
\tilde{K}(\infty, y, \gamma) \geq \tilde{C} y, \quad \forall y=1,2, \ldots,
$$

for some $C, \tilde{C}>0$. Then the gel solution satisfies (3.31).

Theorem 3.10. Let $\gamma \in G$. Assume the kernel satisfies

$$
K(x, y, \gamma) \leq C\left[x y^{a}+x^{a} y\right], \quad \forall x, y=1,2, \ldots,
$$

and

$$
\tilde{K}(\infty, y, \gamma) \geq \tilde{C} y^{a}, \quad \forall y=1,2, \ldots,
$$

for some $C, \tilde{C}>0$ and $a \in[0,1]$. If the sol solution is such that

$$
\lim _{y \rightarrow \infty}\left[y \sup _{s \in[u, t]} \nu(s, y, \gamma)\right]=0, \quad \forall t>u \geq 0: \nu(u+, \infty, \gamma)>0,
$$

then the gel solution satisfies (3.31).

The proof of the theorems is based on the following lemma.

Lemma 3.11. If

$$
\nu(u+, \infty, \gamma)>0, \quad \text { for some } u \geq 0,
$$

implies

$$
\lim _{k \rightarrow \infty} \int_{u}^{t} S(k, \gamma, \nu(s)) d s=0, \quad \forall t>u,
$$

then the gel solution satisfies (3.31). 
Proof. It follows from (3.17) that

$$
\begin{aligned}
\nu(t, \infty, \gamma)= & \nu(\tau(\gamma), \infty, \gamma)+\int_{\tau(\gamma)}^{t} \nu(s, \infty, \gamma) \sum_{y=1}^{\infty} \tilde{K}(\infty, y, \gamma) \nu(s, y, \gamma) d s+ \\
& \lim _{k \rightarrow \infty} \int_{\tau(\gamma)}^{t} S(k, \gamma, \nu(s)) d s, \quad \forall t \geq \tau(\gamma) .
\end{aligned}
$$

If $\nu(\tau(\gamma)+, \infty, \gamma)>0$, then (3.31) follows from 3.39) and 3.38). It follows from 3.39 that

$$
\nu(\tau(\gamma)+, \infty, \gamma)=\nu(\tau(\gamma), \infty, \gamma)+\lim _{\delta \rightarrow 0} \lim _{k \rightarrow \infty} \int_{\tau(\gamma)}^{\tau(\gamma)+\delta} S(k, \gamma, \nu(s)) d s
$$

If $\nu(\tau(\gamma)+, \infty, \gamma)=0$, then 3.39 and 3.40 imply

$$
\begin{aligned}
\nu(t, \infty, \gamma)= & \int_{\tau(\gamma)}^{t} \nu(s, \infty, \gamma) \sum_{y=1}^{\infty} \tilde{K}(\infty, y, \gamma) \nu(s, y, \gamma) d s+ \\
& \lim _{\delta \rightarrow 0} \lim _{k \rightarrow \infty} \int_{\tau(\gamma)+\delta}^{t} S(k, \gamma, \nu(s)) d s, \quad \forall t>\tau(\gamma),
\end{aligned}
$$

so that (3.31) follows from 3.33 and 3.38 .

Proof of Theorem 3.9. The theorem follows from Lemma 3.11. Indeed, (3.37) implies

$$
\int_{u}^{t}\left[\sum_{y=1}^{\infty} \tilde{K}(\infty, y, \gamma) \nu(s, y, \gamma)\right] d s<\infty, \quad \forall t>u,
$$

according to (3.14). It follows from (3.41) and (3.34) that

$$
\int_{u}^{t}\left[\sum_{y=1}^{\infty} y \nu(s, y, \gamma)\right] d s<\infty, \quad \forall t>u .
$$

Thus, 3.38 is a consequence of Lemma 3.3 .

Proof of Theorem 3.10. The theorem follows from Lemma 3.11. Indeed, 3.37) implies

$$
\int_{u}^{t}\left[\sum_{y=1}^{\infty} y^{a} \nu(s, y, \gamma)\right] d s<\infty, \quad \forall t>u
$$

according to (3.14) and (3.35). Thus, (3.38) is a consequence of (3.36) and Lemma 3.4.

\subsubsection{Continuity}

It follows from (3.1) that the functions $\nu(t, x, \gamma)$ are continuous in $t$, for any finite $x$ and $\gamma \in G$.

Here we provide sufficient conditions for the continuity of $\nu(t, \infty, \gamma)$. 
Lemma 3.12. If the sol solution is such that

$$
\int_{0}^{t} \underset{k}{\limsup } S(k, \gamma, \nu(s)) d s<\infty, \quad \forall t \geq 0,
$$

then $\nu(t, \infty, \gamma)$ is continuous in $t$.

Note that

$$
\int_{0}^{t} \liminf _{k \rightarrow \infty} S(k, \gamma, \nu(s)) d s<\infty, \quad \forall t \geq 0,
$$

according to 3.29 and 3.15 .

Lemma 3.12 is an immediate consequence of the following slightly more general result.

Lemma 3.13. If

$$
\int_{t-\varepsilon}^{t} \limsup _{k} S(k, \gamma, \nu(s)) d s<\infty, \quad \text { for } t>0 \text { and some } \varepsilon \in(0, t),
$$

then $\nu(t-, \infty, \gamma)=\nu(t, \infty, \gamma)$. If

$$
\int_{t}^{t+\varepsilon} \limsup _{k} S(k, \gamma, \nu(s)) d s<\infty, \quad \text { for some } \varepsilon>0
$$

then $\nu(t, \infty, \gamma)=\nu(t+, \infty, \gamma)$.

Proof. It follows from (3.17) that

$$
\nu(t, \infty, \gamma)=\nu(t-, \infty, \gamma)+\lim _{\delta \rightarrow 0} \lim _{k \rightarrow \infty} \int_{t-\delta}^{t} S(k, \gamma, \nu(s)) d s, \quad \forall t>0
$$

and

$$
\nu(t+, \infty, \gamma)=\nu(t, \infty, \gamma)+\lim _{\delta \rightarrow 0} \lim _{k \rightarrow \infty} \int_{t}^{t+\delta} S(k, \gamma, \nu(s)) d s, \quad \forall t \geq 0
$$

Since

$$
\lim _{k \rightarrow \infty} \int_{u}^{t} S(k, \gamma, \nu(s)) d s \leq \int_{u}^{t} \limsup _{k \rightarrow \infty} S(k, \gamma, \nu(s)) d s \quad \forall 0 \leq u \leq t,
$$

the assertions follow.

\subsection{Spatially homogeneous case}

Let $|G|$ denote the size of the grid. In the case $|G|=1$, when all particles are located at the same site, the sol equations (3.7) are sufficient to describe the evolution of $\nu$, since

$$
\nu(t, \infty)=1-\sum_{k=1}^{\infty} \nu(t, k) .
$$

In the case $|G|>1$ (with $\kappa>0$ ), equations for $\nu(t, \infty, \gamma), \gamma \in G$, are necessary, since there is mass exchange between different sites. However, even in the spatially homogeneous case the gel equation (3.16) provides additional insight into the gelation phenomenon. 


\subsubsection{Modified coagulation equations}

Here we derive some versions of the limiting equation (2.14) that have been previously studied in the literature.

\section{Weak equations}

Equation (2.14) holds, in particular, for $\varphi \in \mathcal{C}_{c}(\mathcal{Z})$. It takes the form (cf. $(2.7)-(2.9),(3.42)$ )

$$
\begin{gathered}
\langle\varphi, X(t)\rangle=\left\langle\varphi, \nu_{0}\right\rangle+\frac{1}{2} \int_{0}^{t} \int_{\mathcal{Z}} \int_{\mathcal{Z}} \frac{K(x, y)}{x y} \times \\
{[(x+y) \varphi(x+y)-x \varphi(x)-y \varphi(y)] X(s, d x) X(s, d y) d s} \\
-\int_{0}^{t}\left(\int_{\mathcal{Z}} \varphi(x)\left[\lim _{y \rightarrow \infty} \frac{K(x, y)}{y}\right] X(s, d x)\right)[1-X(s, \mathcal{Z})] d s .
\end{gathered}
$$

Introducing the notations $x C(t, d x)=X(t, d x)$ and $\psi(x)=x \varphi(x)$, one obtains from 3.43

$$
\begin{aligned}
& \langle\psi, C(t)\rangle=\left\langle\psi, C_{0}\right\rangle+ \\
& \quad \frac{1}{2} \int_{0}^{t} \int_{\mathcal{Z}} \int_{\mathcal{Z}} K(x, y)[\psi(x+y)-\psi(x)-\psi(y)] C(s, d x) C(s, d y) d s \\
& \quad-\int_{0}^{t}\left(\int_{\mathcal{Z}} \psi(x)\left[\lim _{y \rightarrow \infty} \frac{K(x, y)}{y}\right] C(s, d x)\right)\left[1-\int_{\mathcal{Z}} x C(s, d x)\right] d s,
\end{aligned}
$$

which is a discrete version of the "modified Smoluchowski equation" in [13, Eq. (2.5)].

If the kernel has the form $K(x, y)=f(x) y$, for sufficiently large $y$, where $f(x)=M x$, for some $M>0$ and sufficiently large $x$, then (3.44) takes the form

$$
\begin{aligned}
& \langle\psi, C(t)\rangle=\left\langle\psi, C_{0}\right\rangle+ \\
& \quad \frac{1}{2} \int_{0}^{t} \int_{\mathcal{Z}} \int_{\mathcal{Z}} K(x, y)[\psi(x+y)-\psi(x)-\psi(y)] C(s, d x) C(s, d y) d s \\
& \quad-\int_{0}^{t}\left(\int_{\mathcal{Z}} \psi(x) f(x) C(s, d x)\right)\left[1-\int_{\mathcal{Z}} x C(s, d x)\right] d s,
\end{aligned}
$$

which is a discrete version of the "modification of Smoluchowski's equation" in [23, Eq. (2.6)]. A uniqueness result for that equation is established in [23, Th. 2.3].

\section{Strong equations}

Equations (3.7) take the form (cf. (3.42)

$$
\begin{aligned}
& \frac{\partial}{\partial t} \nu(t, k)=\frac{k}{2} \sum_{x=1}^{k-1} \frac{K(x, k-x)}{x(k-x)} \nu(t, x) \nu(t, k-x)- \\
& \nu(t, k) \sum_{x=1}^{\infty} \frac{K(x, k)}{x} \nu(t, x)-\nu(t, k) \tilde{K}(\infty, k)\left[1-\sum_{x=1}^{\infty} \nu(t, x)\right]
\end{aligned}
$$


and, with the notations $\nu(t, k)=k c(t, k)$,

$$
\begin{aligned}
\frac{\partial}{\partial t} c(t, k) & =\frac{1}{2} \sum_{x=1}^{k-1} K(x, k-x) c(t, x) c(t, k-x)- \\
c(t, k) & \sum_{x=1}^{\infty} K(x, k) c(t, x)-c(t, k) \tilde{K}(\infty, k)\left[1-\sum_{x=1}^{\infty} x c(t, x)\right] .
\end{aligned}
$$

If the kernel has the form

$$
K(x, y)=x y^{a}+x^{a} y,
$$

then the negative terms on the right-hand side of (3.46) are (in the case $a \in[0,1)$ )

$$
\begin{aligned}
& c(t, k) k^{a} \sum_{x=1}^{\infty} x c(t, x)+ \\
& \quad c(t, k) k \sum_{x=1}^{\infty} x^{a} c(t, x)+c(t, k) k^{a}\left[1-\sum_{x=1}^{\infty} x c(t, x)\right]
\end{aligned}
$$

and (in the case $a=1$ )

$$
2 c(t, k) k \sum_{x=1}^{\infty} x c(t, x)+2 c(t, k) k\left[1-\sum_{x=1}^{\infty} x c(t, x)\right] .
$$

According to (3.47), 3.48), one obtains from (3.46) (in the case $a \in[0,1)$ )

$$
\begin{aligned}
& \frac{\partial}{\partial t} c(t, k)= \\
& \quad \frac{1}{2} \sum_{x=1}^{k-1} K(x, k-x) c(t, x) c(t, k-x)-c(t, k) k \sum_{x=1}^{\infty} x^{a} c(t, x)-c(t, k) k^{a}
\end{aligned}
$$

and (in the case $a=1$ )

$$
\frac{\partial}{\partial t} c(t, k)=\frac{1}{2} \sum_{x=1}^{k-1} K(x, k-x) c(t, x) c(t, k-x)-2 c(t, k) k .
$$

Equations (3.49) and (3.50) are discrete versions of the "modified coagulation equation" in [7, Eq. (1.10)].

\subsubsection{Multiplicative kernel}

Here we illustrate some of the results in the special case (1.8), which has been extensively studied in the literature. 


\section{Properties of the solution}

The sol equations (3.7) take the form (cf. 3.45)

$$
\frac{\partial}{\partial t} \nu(t, k)=\frac{k}{2} \sum_{x=1}^{k-1} \nu(t, x) \nu(t, k-x)-k \nu(t, k) .
$$

The unique solution of (3.51), with monodisperse initial conditions, is

$$
\nu(t, k)=\frac{k^{k-1}}{k !} t^{k-1} e^{-k t}, \quad t \geq 0 .
$$

Using Stirling's formula

$$
\sqrt{2 \pi} k^{k+\frac{1}{2}} e^{-k+\frac{1}{12 k+1}}<k !<\sqrt{2 \pi} k^{k+\frac{1}{2}} e^{-k+\frac{1}{12 k}}
$$

one obtains

$$
\begin{aligned}
\frac{1}{\sqrt{2 \pi} t e^{\frac{1}{12 k}}} k^{-\frac{3}{2}} e^{-k(t-1-\log t)}< \\
\nu(t, k)<\frac{1}{\sqrt{2 \pi} t e^{\frac{1}{12 k+1}} k^{-\frac{3}{2}} e^{-k(t-1-\log t)} .}
\end{aligned}
$$

Note that the function $f(t)=t-1-\log t$ satisfies $f^{\prime}(t)=1-\frac{1}{t}$ and

$$
f(t)>0, \quad \forall t \neq 1, \quad f(1)=0 .
$$

In particular, it follows that the moments

$$
m_{\varepsilon}(t):=\sum_{y=1}^{\infty} y^{\varepsilon} \nu(t, y), \quad \varepsilon \geq 0,
$$

remain finite for $t \geq 0$, if $\varepsilon<\frac{1}{2}$, while having a singularity at $t=1$, if $\varepsilon \geq \frac{1}{2}$. Furthermore, it is known that (cf., e.g., [6, p.274], [29, p.922], [25, p.377])

$$
\begin{gathered}
1=\exp (t \nu(t, \infty))(1-\nu(t, \infty)), \\
\lim _{t \searrow 1} \frac{d}{d t} \nu(t, \infty)=2, \\
m_{1}(t)=\frac{1-\nu(t, \infty)}{1-t+t \nu(t, \infty)}
\end{gathered}
$$

and

$$
m_{1}(t) \sim \frac{1}{|1-t|} \quad \text { as } \quad t \rightarrow 1
$$




\section{Gel equation}

Note that

$$
\int_{u}^{t}\left[\sum_{y=1}^{\infty} y \nu(s, y)\right] d s=\infty \quad \text { if } \quad 1 \in[u, t],
$$

according to 3.59 . Thus, 3.20 does not hold and Lemma 3.3 can not be used. However, $\nu(s, k)$ has the order $k^{-\frac{3}{2}}$ for $s=1$ and lower order for $s \neq 1$, according to 3.54). Thus, Lemma 3.5 implies

$$
\sup _{k} S(k, \nu(1))<\infty, \quad \lim _{k \rightarrow \infty} S(k, \nu(s))=0 \quad \forall s \neq 1
$$

where (cf. (3.13))

$$
S(k, \mu)=\sum_{x=1}^{k-1} \mu(x) \sum_{y=k-x}^{\infty} \frac{K(x, y)}{y} \mu(y) .
$$

Moreover, since (cf. 3.52)

$$
\nu^{\prime}(t, k)=\frac{k^{k-1}}{k !} t^{k-2} e^{-k t}[(k-1)-k t]
$$

one obtains

$$
\nu(t, k) \leq \nu_{\max }(k)=\nu((k-1) / k, k)=\frac{(k-1)^{k-1}}{k !} e^{-(k-1)}, \quad \forall t \geq 0,
$$

and, using (3.53)

$$
\frac{1}{\sqrt{2 \pi} e^{\frac{1}{12(k-1)}} k \sqrt{k-1}}<\nu_{\max }(k)<\frac{1}{\sqrt{2 \pi} e^{\frac{1}{12(k-1)+1}} k \sqrt{k-1}} .
$$

Thus,

$$
\nu(t, k) \leq 2 k^{-\frac{3}{2}}, \quad \forall k=1,2, \ldots, \quad t \geq 0,
$$

and it follows from Lemma 3.5 that

$$
\int_{0}^{t} \sup _{k} S(k, \nu(s)) d s<\infty, \quad \forall t \geq 0 .
$$

Finally, Lemma 3.7 implies

$$
\liminf _{k \rightarrow \infty} S(k, \nu(1))>0
$$

According to (3.61) and Lemma 3.12, the gel solution $\nu(t, \infty)$ is continuous. Moreover, the gel production term vanishes, since

$$
\lim _{k \rightarrow \infty} \int_{0}^{t} S(k, \nu(s)) d s=\int_{0}^{t} \lim _{k \rightarrow \infty} S(k, \nu(s)) d s=0 .
$$


The gel equation (3.16) takes the form

$$
\nu(t, \infty)=\nu(0, \infty)+\int_{0}^{t} \nu(s, \infty) m_{1}(s) d s, \quad \forall t \geq 0 .
$$

Note that 3.56 and 3.58 imply

$$
\frac{d}{d t} \nu(t, \infty)=\frac{\nu(t, \infty)(1-\nu(t, \infty))}{1-t+t \nu(t, \infty)}=\nu(t, \infty) m_{1}(t)
$$

which is consistent with equation (3.62). Moreover, one obtains

$$
\lim _{t \searrow 1} \nu(t, \infty) m_{1}(t)=2,
$$

according to 3.57$)$ and $(3.59)$.

\subsubsection{Active and passive gel}

Here we consider kernels of the form

$$
K(x, y)=\frac{1}{2}\left[x^{a} y^{b}+x^{b} y^{a}\right], \quad 1 \geq a \geq b \geq 0, \quad a+b>1,
$$

which have been frequently studied in the literature. We assume that the initial condition is such that $\tau>0$ (cf. (3.32)). Using the results concerning the gel equation and, in particular, the gel production term, we discuss (on a heuristic level) the behavior of the sol solution.

Let the sol solution be such that (cf. (3.60)

$$
\sup _{t \geq 0} \sup _{k} S(k, \nu(t))<\infty
$$

and

$$
\exists \lim _{k \rightarrow \infty} S(k, \nu(t)), \quad \forall t \geq 0 .
$$

The gel equation (3.16) implies (except for $t=\tau$ )

$$
\frac{d}{d t} \nu(t, \infty)=\nu(t, \infty) \sum_{y=1}^{\infty} \tilde{K}(\infty, y) \nu(t, y)+\lim _{k \rightarrow \infty} S(k, \nu(t))
$$

Note that (cf. (3.3)

$$
\tilde{K}(\infty, y)=\left\{\begin{array}{cl}
y, & \text { if } 1=a=b \\
\frac{1}{2} y^{b}, & \text { if } 1=a>b \\
0, & \text { if } 1>a \geq b
\end{array}\right.
$$

According to Lemma 3.5, condition (3.64) is satisfied if

$$
\nu(t, k) \leq C k^{\beta}, \quad \forall k=1,2, \ldots, \quad t \geq 0,
$$


for some $C>0$ and

$$
\beta=-\frac{a+b+1}{2}
$$

Correspondingly, moments 3.55 of the order

$$
\varepsilon<\frac{a+b-1}{2}
$$

remain finite. We refer to [33, p.594], [16, p.553] and [28, p.792] concerning the "critical" exponent (3.68.

For $t<\tau$, equation (3.65) implies (cf. (3.32), 3.33)

$$
\lim _{k \rightarrow \infty} S(k, \nu(t))=0 .
$$

Thus, the solution should decay faster than algebraically with exponent 3.68).

At $t=\tau$, one might expect that the solution reaches algebraic growth with exponent (3.68). Correspondingly, moments 3.55 of the order

$$
\varepsilon \geq \frac{a+b-1}{2}
$$

become infinite.

For $t>\tau$, the behavior is completely different in the cases of active and passive gel.

\section{Active gel case}

In the active gel case $(a=1)$, the first term in (3.65) takes control at $t=\tau$ (cf. (3.31)). Moments $m_{b}(t)$ (cf. (3.55) ) become finite and, as a consequence, $(3.69)$ holds. Thus, the behavior of the solution is the same as before $\tau$. In fact, if the solution satisfies the growth condition (3.67), then the finiteness of $m_{b}(t)$ implies $\beta<-b-1$, which is stronger than $\beta<-\frac{b}{2}-1$ (cf. 3.68). The gel equation (3.65) takes the form

$$
\frac{d}{d t} \nu(t, \infty)=\nu(t, \infty) \sum_{y=1}^{\infty} \tilde{K}(\infty, y) \nu(t, y)
$$

For the kernel (3.63) with $a=1$ and $b \in(0,1)$, one obtains (cf. 3.66), 3.55)

$$
\frac{d}{d t} \nu(t, \infty)=\frac{1}{2} \nu(t, \infty) m_{b}(t)
$$

\section{Passive gel case}

In the passive gel case $(a<1)$, the first term in 3.65 disappears and gelation is controlled by the second term. The gel equation (3.65) takes the form

$$
\frac{d}{d t} \nu(t, \infty)=\lim _{k \rightarrow \infty} S(k, \nu(t))
$$


so that

$$
\lim _{k \rightarrow \infty} S(k, \nu(t))>0 .
$$

Thus, the solution keeps to be of the order (3.68), according to Lemma 3.5 and Lemma 3.7. For the kernel $(3.63)$ with $b=a$ and $a \in(0.5,1)$, the critical order is $\beta=-a-\frac{1}{2}$. Correspondingly, moments of the order $\varepsilon<a-\frac{1}{2}$ stay finite, while moments of the order $\varepsilon \geq a-\frac{1}{2}$ stay infinite. In particular, one concludes that $m_{a}(t)=\infty$. Note that the moments $m_{\varepsilon}(t), \varepsilon>0$, grow monotonically, which can be derived from the weak form of the equation.

\subsubsection{Special initial conditions}

Here we consider kernels of the form $(3.63)$ and discuss initial conditions leading to $\tau=0$ (cf. (3.32).

\section{Slowly decaying initial distributions}

In the case of the multiplicative kernel $(1.8)$ it is known that [23, Th. 2.8]

$$
\tau=\frac{1}{m_{1}(0)} .
$$

Thus, $m_{1}(0)=\infty$ is a necessary and sufficient condition for $\tau=0$, or, in other words, sufficiently slow decay of $\nu(0, x)$ in $x$ leads to immediate gelation.

In the general case (3.63), it is of interest to consider initial distributions satisfying

$$
\nu(0, k) \geq C k^{\beta}, \quad \forall k=1,2, \ldots,
$$

for some $C>0$ and $\beta$ such that

$$
-\frac{a+b+1}{2}<\beta<-1 .
$$

According to Lemma 3.7, condition 3.72 implies

$$
\lim _{k \rightarrow \infty} S(k, \nu(0))=\infty
$$

so that (3.64) does not hold. In the active gel case $(a=1)$, the behavior for $t>0$ seems to remain the same as in the case $t>\tau>0$, discussed before. In the passive gel case $(a<1)$, equation (3.71) would suggest an infinite slope of the gel solution, i.e. $\nu^{\prime}(0+, \infty)=\infty$. However, even a rigorous conclusion about continuity would need further information about the sol solution (cf. Lemma 3.12.

\section{Initial gel}

Consider the case $\nu(0, \infty)>0$ (cf. Remark 3.2). In the active gel case $(a=1)$, the gel mass starts growing immediately. Its slope depends on the corresponding moment, according to equation (3.70). Note that this moment should be integrable in any neighborhood of $t=0$ (compare 
this with $(3.59)$ ). In the passive gel case $(a<1)$, the gel mass may remain constant and start growing later (dependent on the sol component of the initial condition). So, having in mind the passive gel case, it might be appropriate to define the gelation time as

$$
\tau(\gamma):=\inf \{t>0: \nu(t, \infty, \gamma)>\nu(0, \infty, \gamma)\},
$$

instead of (3.32). In the active gel case both definitions are equivalent (except the trivial case $\nu(0, \infty)=1)$.

An interesting aspect of the initial gel case is that even the consideration of non-gelling kernels makes sense. In the passive gel case (e.g., $K(x, y)=1$ ), the sol and the gel develop independently. However, in the active gel case the initial gel starts growing immediately. The linear kernel $K(x, y)=x+y$ is an example of a non-gelling kernel, for which the gel is active. One obtains $\tilde{K}(\infty, y)=1$ and equation 3.70 takes the form

$$
\frac{d}{d t} \nu(t, \infty)=\nu(t, \infty)[1-\nu(t, \infty)]
$$

Note that the sol equations are modified in the initial gel case.

\subsection{Comments}

Here we give some comments concerning the two spatially inhomogeneous gelation models mentioned in the introduction.

\subsubsection{The van Dongen model}

The sol equations (3.7), with the notations $\nu(t, k, \gamma)=k c(t, k, \gamma)$, take the form

$$
\begin{aligned}
& \frac{\partial}{\partial t} c(t, k, \gamma)=\sum_{\alpha \neq \gamma} \kappa(k, \alpha, \gamma) c(t, k, \alpha)-c(t, k, \gamma) \sum_{\beta \neq \gamma} \kappa(k, \gamma, \beta)+ \\
& \quad \frac{1}{2} \sum_{x=1}^{k-1} K(x, k-x, \gamma) c(t, x, \gamma) c(t, k-x, \gamma)- \\
& \quad c(t, k, \gamma) \sum_{x=1}^{\infty} K(x, k, \gamma) c(t, x, \gamma)-c(t, k, \gamma) \tilde{K}(\infty, k, \gamma) \nu(t, \infty, \gamma)
\end{aligned}
$$

which is a spatially discrete version of (1.11), when the multiplicative kernel (1.8) is chosen. Moreover, equation (3.31) holds, according to Theorem 3.9, and provides a spatially discrete version of 1.12 .

\subsubsection{Formal extensions of Smoluchowski's coagulation equation}

Note that (3.73) is a spatially discrete version of $(1.4)$, when

$$
\tilde{K}(\infty, k, \gamma)=0
$$


In this case, equation $(3.16)$ can be formally transformed into

$$
\frac{\partial}{\partial t} \nu(t, \infty, \gamma)=\lim _{k \rightarrow \infty}\left[\sum_{x=1}^{k-1} x c(t, x, \gamma) \sum_{y=k-x}^{\infty} K(x, y, \gamma) c(t, y, \gamma)\right],
$$

which is a spatially discrete version of (1.9), (1.10) (without the gradient term), when the multiplicative kernel (1.8) is chosen.

However, condition (3.74) is not fulfilled for the kernel (1.8). Before commenting on this point, we illustrate the situation in the spatially homogeneous case. When skipping the term containing $\tilde{K}$, equations $(3.73$ take the form

$$
\frac{\partial}{\partial t} \bar{c}(t, k)=\frac{1}{2} \sum_{x=1}^{k-1} x(k-x) \bar{c}(t, x) \bar{c}(t, k-x)-k \bar{c}(t, k) \sum_{x=1}^{\infty} x \bar{c}(t, x) .
$$

This is Smoluchowski's coagulation equation (1.5) formally extended to the kernel $(1.8)$. It is known that the solution of (3.76), with monodisperse initial conditions, satisfies (cf. (3.52)

$$
\bar{c}(t, k)=\frac{1}{k} \nu(1, k) \frac{1}{t}, \quad t \geq 1 .
$$

According to (3.77), growth properties (with respect to $k$ ) at $t=1$ remain valid for $t>1$. This behavior of the solution reminds the passive gel case discussed in Section 3.3.3.

Equation (3.76) is "wrong" in the sense that its solution does not approximate the corresponding Marcus-Lushnikov process. In general, various coagulation kernels are derived from certain assumptions on the underlying physical system. Smoluchowski derived his equation with the particular (non-gelling) kernel (1.6) starting from a system of diffusing spherical particles. Thus, it might be more appropriate to call equation (3.76) a "formal" Smoluchowski equation. Rigorous results concerning the transition from stochastic particle systems to the solution (3.77) would need some truncation of the kernel dependent on the number of monomers in the system (cf. [1, Conjecture 3.6]). Due to this truncation, the gel would not interact with the sol, thus becoming "passive". This explains why the solution of the formal extension of Smoluchowski's coagulation equation to the multiplicative kernel behaves like a solution in the passive gel case.

Turning to the model (1.9), (1.10), the form of the gel production term can be explained now by analogy with the passive gel case. However, in the spatially inhomogeneous situation the spatial behavior of the gel has to be described, in addition to its growth properties. Simply adding a diffusion term seems to be another formal extension of Smoluchowski's coagulation equation. It is not clear if this model is of any practical relevance, since the gel would be expected to behave randomly, even if a truncation of the kernel was used. The asymptotic behavior of the gel is determined by the assumptions on the diffusion coefficients. For non-vanishing $D(k)$, a stochastic limit was predicted in [27]. We also refer to the corresponding discussion in [25]. 


\section{Proofs}

\subsection{Proof of Theorem 2.1}

\section{Properties of the processes}

Note that (cf. (2.1), 1.3) )

$$
\mathbb{P}\left(X^{N} \in \mathcal{D}\left([0, \infty), \mathcal{P}\left(\mathcal{Z}^{\prime}\right)\right)\right)=1 .
$$

Consider the generator (cf. (2.2)-(2.4)

$$
\mathcal{A}^{N} \Phi(\mu)=\int_{E^{N}}[\Phi(\nu)-\Phi(\mu)] \lambda^{N}(\mu, d \nu)
$$

and test functions of the form

$$
\Phi(\mu)=\langle\varphi, \mu\rangle=\frac{1}{N} \sum_{i=1}^{n} x_{i} \varphi\left(x_{i}, \alpha_{i}\right) .
$$

Note that $|\Phi(\mu)| \leq\|\varphi\|_{\infty}$. The usual starting point for deriving a limiting equation is the martingale representation

$$
\left\langle\varphi, X^{N}(t)\right\rangle=\left\langle\varphi, X^{N}(0)\right\rangle+\int_{0}^{t} \mathcal{A}^{N} \Phi\left(X^{N}(s)\right) d s+M^{N}(\varphi, t) .
$$

Helpful properties are

$$
\begin{gathered}
\mathbb{E} \sup _{s \leq t}\left|M^{N}(\varphi, s)\right| \leq 4 \mathbb{E} M^{N}(\varphi, t)^{2} \\
\mathbb{E} M^{N}(\varphi, t)^{2}=\mathbb{E} \int_{0}^{t}\left[\mathcal{A}^{N} \Phi^{2}-2 \Phi \mathcal{A}^{N} \Phi\right]\left(X^{N}(s)\right) d s \\
{\left[\mathcal{A}^{N} \Phi^{2}-2 \Phi \mathcal{A}^{N} \Phi\right](\mu)=\int_{E^{N}}[\langle\varphi, \nu\rangle-\langle\varphi, \mu\rangle]^{2} \lambda^{N}(\mu, d \nu)}
\end{gathered}
$$

and (for any $k \geq 0$ )

$$
\begin{aligned}
\int_{E^{N}} & {[\langle\varphi, \nu\rangle-\langle\varphi, \mu\rangle]^{k} \lambda^{N}(\mu, d \nu)=} \\
& \sum_{i=1}^{n} \sum_{\beta} \kappa\left(x_{i}, \alpha_{i}, \beta\right)\left[\left\langle\varphi, J_{1}(\mu, i, \beta)\right\rangle-\langle\varphi, \mu\rangle\right]^{k}+ \\
& \frac{1}{2 N} \sum_{1 \leq i \neq j \leq n} \delta_{\alpha_{i}, \alpha_{j}} K\left(x_{i}, x_{j}, \alpha_{i}\right)\left[\left\langle\varphi, J_{2}(\mu, i, j)\right\rangle-\langle\varphi, \mu\rangle\right]^{k} \\
= & \frac{1}{N^{k}} \sum_{i=1}^{n} \sum_{\beta} \kappa\left(x_{i}, \alpha_{i}, \beta\right) x_{i}^{k}\left[\varphi\left(x_{i}, \beta\right)-\varphi\left(x_{i}, \alpha_{i}\right)\right]^{k}+ \\
& \frac{1}{2 N^{k+1}} \sum_{1 \leq i \neq j \leq n} \delta_{\alpha_{i}, \alpha_{j}} K\left(x_{i}, x_{j}, \alpha_{i}\right) \times \\
& {\left[\left(x_{i}+x_{j}\right) \varphi\left(x_{i}+x_{j}, \alpha_{i}\right)-x_{i} \varphi\left(x_{i}, \alpha_{i}\right)-x_{j} \varphi\left(x_{j}, \alpha_{j}\right)\right]^{k} . }
\end{aligned}
$$




\section{Estimates for the generator}

Lemma 4.1. If $\varphi$ has the form (2.15), then

$$
|(x+y) \varphi(x+y, \alpha)-x \varphi(x, \alpha)-y \varphi(y, \alpha)| \leq 4\|\varphi\| \bar{x}(\varphi) .
$$

Proof. If $x \leq \bar{x}(\varphi)$ and $y>\bar{x}(\varphi)$, then the left-hand side of (4.7) takes the form $\mid x c_{0}(\varphi, \alpha)-$ $x \varphi(x, \alpha) \mid$. Other cases are treated analogously.

Lemma 4.2. Assume

$$
\kappa(x, \alpha, \beta) \leq C_{\kappa}, \quad \forall \alpha, \beta \in G, \quad x=1,2, \ldots,
$$

for some $C_{\kappa}>0$, and (2.12). Then

$$
\sup _{N} \sup _{\mu \in E^{N}}\left|\mathcal{A}^{N} \Phi(\mu)\right|<\infty,
$$

for any $\varphi$ satisfying 2.15).

Proof. One obtains from 4.1), 4.6 (with $k=1$ ) and Lemma 4.1 that

$$
\begin{aligned}
\left|\mathcal{A}^{N} \Phi(\mu)\right| \leq & \frac{1}{N} \sum_{i=1}^{n} \sum_{\beta} \kappa\left(x_{i}, \alpha_{i}, \beta\right) x_{i}\left|\varphi\left(x_{i}, \beta\right)-\varphi\left(x_{i}, \alpha_{i}\right)\right|+ \\
& \frac{1}{2 N^{2}} \sum_{i, j=1}^{n} \delta_{\alpha_{i}, \alpha_{j}} K\left(x_{i}, x_{j}, \alpha_{i}\right) \times \\
& \left|\left(x_{i}+x_{j}\right) \varphi\left(x_{i}+x_{j}, \alpha_{i}\right)-x_{i} \varphi\left(x_{i}, \alpha_{i}\right)-x_{j} \varphi\left(x_{j}, \alpha_{j}\right)\right| \\
\leq & 2\|\varphi\| C_{\kappa}|G|+2\|\varphi\| \bar{x}(\varphi) C_{K},
\end{aligned}
$$

and the assertion follows.

\section{Estimates for the martingale term}

Lemma 4.3. Assume 2.10 and 2.12). Then

$$
\lim _{N \rightarrow \infty} \mathbb{E} \sup _{s \leq t}\left|M^{N}(\varphi, s)\right|=0
$$

for any $\varphi$ satisfying 2.15).

Proof. One obtains from (4.3) - 4.5) and 4.6) (with $k=2$ )

$$
\begin{aligned}
& \mathbb{E} \sup _{s \leq t}\left|M^{N}(\varphi, s)\right| \leq \\
& 4 t \sup _{\mu \in E^{N}} \frac{1}{N^{2}} \sum_{i=1}^{n} \sum_{\beta \in G} \kappa\left(x_{i}, \alpha_{i}, \beta\right) x_{i}^{2}\left[\varphi\left(x_{i}, \beta\right)-\varphi\left(x_{i}, \alpha_{i}\right)\right]^{2}+ \\
& 2 t \sup _{\mu \in E^{N}} \frac{1}{N^{3}} \sum_{i, j=1}^{n} K\left(x_{i}, x_{j}, \alpha_{i}\right) \times \\
& {\left[\left(x_{i}+x_{j}\right) \varphi\left(x_{i}+x_{j}, \alpha_{i}\right)-x_{i} \varphi\left(x_{i}, \alpha_{i}\right)-x_{j} \varphi\left(x_{j}, \alpha_{i}\right)\right]^{2} .}
\end{aligned}
$$


Let $\varepsilon>0$ and choose $x(\varepsilon)$ such that

$$
\kappa(x, \alpha, \beta) \leq \varepsilon, \quad \forall x>x(\varepsilon) .
$$

Then 4.9 and Lemma 4.1 imply

$$
\begin{aligned}
& \mathbb{E} \sup _{s \leq t}\left|M^{N}(\varphi, s)\right| \leq \\
& \quad 16 t\|\varphi\|^{2}|G|\left[C_{\kappa} \frac{x(\varepsilon)}{N}+\varepsilon \frac{1}{N^{2}} \sum_{i=1}^{n} x_{i}^{2}\right]+32 t\|\varphi\|^{2} \bar{x}(\varphi)^{2} C_{K} \frac{1}{N} .
\end{aligned}
$$

Since $\sum_{i=1}^{n} x_{i}=N$, one obtains

$$
\limsup _{N \rightarrow \infty} \mathbb{E} \sup _{s \leq t}\left|M^{N}(\varphi, s)\right| \leq 16 t\|\varphi\|^{2}|G| \varepsilon
$$

and 4.8 follows.

\section{Relative compactness}

Lemma 4.4. The set

$$
\left\{d_{k, \gamma}, \psi_{k, \gamma}: \gamma \in G, k=1,2, \ldots\right\}
$$

of functions (3.4), (3.5) is convergence determining (with respect to weak convergence in $\mathcal{P}\left(\mathcal{Z}^{\prime}\right)$ ).

Proof. According to [9, Lemma 3.4.3], it is sufficient to show that the set 4.10) is separating. From $\left\langle d_{k, \beta}, \mu\right\rangle=\left\langle d_{k, \beta}, \nu\right\rangle$ one obtains $\mu(k, \beta)=\nu(k, \beta)$, and $\left\langle\psi_{k, \beta}, \mu\right\rangle=\left\langle\psi_{k, \beta}, \nu\right\rangle$ for all $k=$ $1,2, \ldots$ implies $\mu(\infty, \beta)=\nu(\infty, \beta)$.

To prove relative compactness of the sequence $\left(X^{N}\right)$ we apply [9, Theorem 3.7.6] with $E=\mathcal{P}\left(\mathcal{Z}^{\prime}\right)$ and the metric (cf. Lemma 4.4)

$$
r(\mu, \nu)=\sum_{k=1}^{\infty} \frac{\min \left(1,\left|\left\langle\varphi_{k}, \mu\right\rangle-\left\langle\varphi_{k}, \nu\right\rangle\right|\right)}{2^{k}}
$$

where $\left(\varphi_{k}\right)$ denote the reordered elements of the set (4.10). The compact containment condition is trivial, since the space $\mathcal{P}\left(\mathcal{Z}^{\prime}\right)$ is compact. The remaining condition to be checked is

$$
\forall T, \varepsilon>0 \quad \exists \delta>0 \quad: \quad \sup _{N} \mathbb{P}\left(w\left(X^{N}, \delta, T\right) \geq \varepsilon\right) \leq \varepsilon
$$

where the modulus of continuity

$$
w(\mu, \delta, T)=\inf _{\left\{t_{i}\right\}} \max _{i} \sup _{s, t \in\left[t_{i-1}, t_{i}\right)} r(\mu(s), \mu(t))
$$

is defined for $\delta, T>0$ and $\mu \in \mathcal{D}([0, \infty), E)$. Here $\left\{t_{i}\right\}$ ranges over all partitions of the form $0=t_{0}<t_{1}<\cdots<t_{n-1}<T \leq t_{n}$ with $\min _{1 \leq i \leq n}\left(t_{i}-t_{i-1}\right)>\delta$ and $n \geq 1$. 
Lemma 4.5. Assume 2.10, 2.12) and let $\varphi$ be of the form 2.15). Then, for any $T, \varepsilon>0$, there exist $\Delta t, N_{0}>0$ such that

$$
\sup _{N \geq N_{0}} \mathbb{P}\left(\sup _{|s-t| \leq \Delta t, t \leq T}\left|\left\langle\varphi, X^{N}(s)\right\rangle-\left\langle\varphi, X^{N}(t)\right\rangle\right| \geq \varepsilon\right) \leq \varepsilon .
$$

Proof. One obtains from 4.2

$$
\left|\left\langle\varphi, X^{N}(t)\right\rangle-\left\langle\varphi, X^{N}(s)\right\rangle\right| \leq\left|M^{N}(\varphi, t)-M^{N}(\varphi, s)\right|+|t-s| c,
$$

where $c:=\sup _{N} \sup _{\mu \in E^{N}}\left|\mathcal{A}^{N} \Phi(\mu)\right|<\infty$, according to Lemma 4.2 . Let $0<\Delta t<\frac{\varepsilon}{2 c} \wedge 1$. It follows from 4.15) that

$$
\begin{aligned}
& \mathbb{P}\left(\sup _{|s-t| \leq \Delta t, t \leq T}\left|\left\langle\varphi, X^{N}(s)\right\rangle-\left\langle\varphi, X^{N}(t)\right\rangle\right| \geq \varepsilon\right) \leq \\
& \mathbb{P}\left(\frac{\varepsilon}{2}+\sup _{|s-t| \leq \Delta t, t \leq T}\left|M^{N}(\varphi, s)-M^{N}(\varphi, t)\right| \geq \varepsilon\right) \\
& \quad \leq \mathbb{P}\left(\sup _{t \leq T+1}\left|M^{N}(\varphi, t)\right| \geq \frac{\varepsilon}{4}\right) \leq \frac{4}{\varepsilon} \mathbb{E} \sup _{t \leq T+1}\left|M^{N}(\varphi, t)\right| .
\end{aligned}
$$

By Lemma 4.3 , the mean value at the right-hand side of 4.16 becomes smaller than $\varepsilon^{2} / 4$ for sufficiently large $N$ so that 4.14 is satisfied.

Lemma 4.6. Assume (2.10) and (2.12). Then, for any $T, \varepsilon>0$, there exist $\Delta t, N_{0}>0$ such that

$$
\sup _{N \geq N_{0}} \mathbb{P}\left(\sup _{|s-t| \leq \Delta t, t \leq T} r\left(X^{N}(s), X^{N}(t)\right) \geq \varepsilon\right) \leq \varepsilon .
$$

Proof. Fix $T, \varepsilon>0$ and choose $L=L(\varepsilon) \geq 0$ such that $\sum_{k=L+1}^{\infty} \frac{1}{2^{k}} \leq \frac{\varepsilon}{2}$. One obtains (cf. 4.11)

$$
\begin{aligned}
& \mathbb{P}\left(\sup _{|s-t| \leq \Delta t, t \leq T} r\left(X^{N}(s), X^{N}(t)\right) \geq \varepsilon\right) \leq \\
& \quad \mathbb{P}\left(\sup _{|s-t| \leq \Delta t, t \leq T} \sum_{k=1}^{L}\left|\left\langle\varphi_{k}, X^{N}(s)\right\rangle-\left\langle\varphi_{k}, X^{N}(t)\right\rangle\right| \geq \frac{\varepsilon}{2}\right) \\
& \quad \leq \sum_{k=1}^{L} \mathbb{P}\left(\sup _{|s-t| \leq \Delta t, t \leq T}\left|\left\langle\varphi_{k}, X^{N}(s)\right\rangle-\left\langle\varphi_{k}, X^{N}(t)\right\rangle\right| \geq \frac{\varepsilon}{2 L}\right) .
\end{aligned}
$$

According to Lemma 4.5, there are $\Delta t, N_{0}>0$ such that 4.14 holds for all $\varphi=\varphi_{k}$ with $k=1, \ldots, L$ (cf. Remark 3.1). Thus, inequality 4.17) follows from 4.18).

For any $T, \varepsilon>0$ and $N$ there exists $\delta_{N}>0$ such that

$$
\mathbb{P}\left(w\left(X^{N}, \delta_{N}, T\right) \geq \varepsilon\right) \leq \varepsilon,
$$


according to [9, Lemma 3.6.2(a)]. Note that $w$ decreases with $\delta$. Thus, condition (4.12) follows from

$$
\forall T, \varepsilon>0 \quad \exists \delta, N_{0}>0 \quad: \quad \sup _{N \geq N_{0}} \mathbb{P}\left(w\left(X^{N}, \delta, T\right) \geq \varepsilon\right) \leq \varepsilon
$$

by choosing $0<\delta<\min \left\{\delta_{1}, \ldots, \delta_{N_{0}-1}\right\}$. Note that (cf. 4.13)

$$
w(\mu, \delta, T) \leq \sup _{|s-t| \leq \Delta t, s \leq T} r(\mu(s), \mu(t)), \quad 0<\delta<\Delta t .
$$

Thus, condition (4.19) is a consequence of 4.20 and Lemma 4.6 .

\section{Characterization of weak limits}

Using (4.2), 4.1) and 4.6) (with $k=1$ ), one obtains (cf. 2.7)

$$
\begin{aligned}
& \left\langle\varphi, X^{N}(t)\right\rangle= \\
& \quad\left\langle\varphi, X^{N}(0)\right\rangle+\int_{0}^{t} \mathcal{G}\left(\varphi, X^{N}(s)\right) d s+M^{N}(\varphi, t)+R^{N}(\varphi, t),
\end{aligned}
$$

where

$$
R^{N}(\varphi, t)=\int_{0}^{t}\left[\mathcal{A}^{N} \Phi\left(X^{N}(s)\right)-\mathcal{G}\left(\varphi, X^{N}(s)\right)\right] d s .
$$

Lemma 4.7. If $\varphi$ satisfies (2.15), then

$$
\lim _{N \rightarrow \infty} \mathbb{E} \sup _{s \leq t}\left|R^{N}(\varphi, s)\right|=0 .
$$

Proof. It follows from (4.1), 4.6) (with $k=1$ ) that

$$
\mathcal{A}^{N} \Phi(\mu)-\mathcal{G}(\varphi, \mu)=-\frac{1}{N^{2}} \sum_{i=1}^{n} x_{i} K\left(x_{i}, x_{i}, \alpha_{i}\right)\left[\varphi\left(2 x_{i}, \alpha_{i}\right)-\varphi\left(x_{i}, \alpha_{i}\right)\right]
$$

and

$$
\left|\mathcal{A}^{N} \Phi(\mu)-\mathcal{G}(\varphi, \mu)\right| \leq 2\|\varphi\| \sup _{x \leq \bar{x}(\varphi), \alpha \in G} K(x, x, \alpha) \frac{1}{N^{2}} \sum_{i=1}^{n} x_{i} .
$$

Thus, one obtains

$$
\lim _{N \rightarrow \infty} \sup _{\mu \in E^{N}}\left|\mathcal{A}^{N} \Phi(\mu)-\mathcal{G}(\varphi, \mu)\right|=0
$$

and 4.23) follows from 4.22.

Lemma 4.8. Assume 2.10, (2.11) and let $\varphi$ be of the form 2.15). Then the mapping

$$
M_{\varphi}: \quad \mathcal{D}\left([0, \infty), \mathcal{P}\left(\mathcal{Z}^{\prime}\right)\right) \rightarrow \mathcal{D}([0, \infty), \mathbb{R})
$$

defined as (cf. (2.14))

$$
M_{\varphi}(\mu)(t)=\langle\varphi, \mu(t)\rangle-\langle\varphi, \mu(0)\rangle-\int_{0}^{t} \mathcal{G}(\varphi, \mu(s)) d s, \quad t \geq 0,
$$

is continuous. 
Proof. This is a consequence of the continuity of $\mathcal{G}(\varphi, \mu)$ with respect to $\mu$ (cf. Remark 2.3).

Lemma 4.9. Assume 2.10, (2.12) and let $\varphi$ be of the form 2.15). Then any limiting point $X$ of the sequence $X^{N}$ satisfies

$$
\mathbb{P}\left(M_{\varphi}(X)=0\right)=1
$$

Proof. Fix $t>0$ and $\varphi$. Since (cf. 4.24, 4.21)

$$
\sup _{s \leq t}\left|M_{\varphi}\left(X^{N}, s\right)\right| \leq \sup _{s \leq t}\left|M^{N}(\varphi, s)\right|+\sup _{s \leq t}\left|R^{N}(\varphi, s)\right|,
$$

it follows from Lemma 4.3 and Lemma 4.7 that

$$
\lim _{N \rightarrow \infty} \mathbb{P}\left(\sup _{s \leq t}\left|M_{\varphi}\left(X^{N}, s\right)\right| \geq \varepsilon\right)=0, \quad \forall \varepsilon>0,
$$

and

$$
\sup _{s \leq t}\left|M_{\varphi}\left(X^{N}, s\right)\right| \Rightarrow 0 \quad \text { as } \quad N \rightarrow \infty
$$

Suppose

$$
X^{N_{l}} \Rightarrow X
$$

for some subsequence. Then Lemma 4.8 implies $M_{\varphi}\left(X^{N_{l}}\right) \Rightarrow M_{\varphi}(X)$ and

$$
d\left(M_{\varphi}\left(X^{N_{l}}\right), 0\right) \Rightarrow d\left(M_{\varphi}(X), 0\right),
$$

where $d$ denotes the Skorokhod metric. On the other hand, 4.25) implies

$$
d\left(M_{\varphi}\left(X^{N}\right), 0\right) \Rightarrow 0 \quad \text { and } \quad d\left(M_{\varphi}(X), 0\right)=0 \quad \text { a.s. }
$$

so that the assertion follows.

As a consequence of Lemma 4.9 , one obtains

$$
\mathbb{P}\left(M_{\varphi_{k}}(X)=0, \forall k\right)=1 .
$$

Assumption (2.13) and (4.26) imply $X(0)=\nu_{0}$ almost surely, so that equation (2.14) is fulfilled for all functions $\left(\varphi_{k}\right)$. Finally, any function of the form (2.15) can be approximated by linear combinations (with rational coefficients) of functions $\left(\varphi_{k}\right)$ in such a way that the corresponding values of $\mathcal{G}$ converge.

\section{Continuity}

Note that

$$
\sup _{t \leq T} r\left(X^{N}(t), X^{N}(t-)\right) \leq \sup _{|s-t| \leq \Delta t, t \leq T} r\left(X^{N}(s), X^{N}(t)\right), \quad \forall \Delta t>0 .
$$


Thus, Lemma 4.6 implies

$$
\sup _{N \geq N_{0}} \mathbb{P}\left(\sup _{t \leq T} r\left(X^{N}(t), X^{N}(t-)\right) \geq \varepsilon\right) \leq \varepsilon,
$$

so that

$$
\sup _{t \leq T} r\left(X^{N}(t), X^{N}(t-)\right) \Rightarrow 0, \quad \forall T>0,
$$

as $N \rightarrow \infty$. An application of [9, Theorem 3.10.2(a)] gives

$$
\mathbb{P}\left(X \in \mathcal{C}\left([0, \infty), \mathcal{P}\left(\mathcal{Z}^{\prime}\right)\right)\right)=1,
$$

for every weak limit $X$ of the sequence $\left(X^{N}\right)$.

This completes the proof of Theorem 2.1.

\subsection{Proof of Lemma 3.3}

Lemma 4.10. If

$$
K(x, y) \leq C x y, \quad \forall x, y=1,2, \ldots, \quad \text { for some } \quad C>0
$$

then

$$
\sum_{x=1}^{k-1} \mu(x) \sum_{y=k-x}^{\infty} \frac{K(x, y)}{y} \mu(y) \leq 2 C\left(\sum_{x=1}^{\infty} x \mu(x)\right) \sum_{x=\min \left(k-k^{\prime}, k^{\prime}+1\right)}^{\infty} x \mu(x),
$$

for any $1 \leq k^{\prime} \leq k-1$.

Proof. One obtains

$$
\begin{aligned}
\frac{1}{C} \sum_{x=1}^{k-1} \mu(x) \sum_{y=k-x}^{\infty} \frac{K(x, y)}{y} \mu(y) \leq \\
\quad \sum_{x=1}^{k^{\prime}} x \mu(x) \sum_{y=k-x}^{\infty} \mu(y)+\sum_{x=k^{\prime}+1}^{k-1} x \mu(x) \sum_{y=k-x}^{\infty} \mu(y) \\
\leq \sum_{x=1}^{\infty} x \mu(x) \sum_{y=k-k^{\prime}}^{\infty} \mu(y)+\sum_{x=k^{\prime}+1}^{\infty} x \mu(x) \sum_{y=1}^{\infty} \mu(y) \\
\leq\left(\sum_{x=1}^{\infty} x \mu(x)\right)\left[\sum_{y=k-k^{\prime}}^{\infty} y \mu(y)+\sum_{x=k^{\prime}+1}^{\infty} x \mu(x)\right]
\end{aligned}
$$

so that the assertion follows. 
Corollary 4.11. If (4.27) and

$$
\sum_{y=1}^{\infty} y \mu(y)<\infty
$$

then

$$
\lim _{k \rightarrow \infty} \sum_{x=1}^{k-1} \mu(x) \sum_{y=k-x}^{\infty} \frac{K(x, y)}{y} \mu(y)=0 .
$$

Proof. Choose $k^{\prime}:=[k / 2]$ (closest integer). Since

$$
\lim _{k \rightarrow \infty} \sum_{x=\min \left(k-k^{\prime}, k^{\prime}+1\right)}^{\infty} x \mu(x)=0,
$$

the assertion follows from Lemma 4.10 .

Since

$$
S(k, \gamma, \nu(s))=\sum_{x=1}^{k-1} \nu(s, x, \gamma) \sum_{y=k-x}^{\infty} \frac{K(x, y, \gamma)}{y} \nu(s, y, \gamma) \leq C \sum_{x=1}^{\infty} x \nu(s, x, \gamma),
$$

assumption 3.20 and the dominated convergence theorem imply

$$
\lim _{k \rightarrow \infty} \int_{u}^{t} S(k, \gamma, \nu(s)) d s=\int_{u}^{t} \lim _{k \rightarrow \infty} S(k, \gamma, \nu(s)) d s .
$$

On the other hand, 3.20 implies $\sum_{y=1}^{\infty} y \nu(s, y, \gamma)<\infty$, for almost all $s \in[u, t]$. Thus, the assertion follows from 4.28 and Corollary 4.11.

\subsection{Proof of Lemma 3.4}

Lemma 4.12. If

$$
\begin{aligned}
K(x, y) \leq & C\left[x y^{a}+x^{a} y\right], \quad \forall x, y=1,2, \ldots, \\
& \text { for some } a \in[0,1], \quad C>0,
\end{aligned}
$$

then

$$
\begin{gathered}
\frac{1}{C} \sum_{x=1}^{k-1} \mu(x) \sum_{y=k-x}^{\infty} \frac{K(x, y)}{y} \mu(y) \leq \\
\sum_{y=1}^{\infty} y^{a} \mu(y)\left[\sup _{x \geq k-k^{\prime}} x \mu(x)+\sum_{y=k-k^{\prime}}^{\infty} \mu(y)\right]+ \\
\sum_{y=k^{\prime}+1}^{\infty} y^{a} \mu(y)\left[2 \sup _{x} x \mu(x)+\sum_{y=1}^{\infty} \mu(y)\right],
\end{gathered}
$$

for any $1 \leq k^{\prime} \leq k-1$. 
Proof. One obtains

$$
\begin{aligned}
& \frac{1}{C} \sum_{x=1}^{k-1} \mu(x) \sum_{y=k-x}^{\infty} \frac{K(x, y)}{y} \mu(y) \leq \\
& \quad \sum_{x=1}^{k-1} x \mu(x) \sum_{y=k-x}^{\infty} y^{a-1} \mu(y)+\sum_{x=1}^{k-1} x^{a} \mu(x) \sum_{y=k-x}^{\infty} \mu(y) \\
&=\sum_{x=1}^{k-1} x \mu(x) \sum_{y=k-x}^{k-1} y^{a-1} \mu(y)+\sum_{x=1}^{k-1} x \mu(x) \sum_{y=k}^{\infty} y^{a-1} \mu(y)+ \\
& \quad \sum_{x=1}^{k^{\prime}} x^{a} \mu(x) \sum_{y=k-x}^{\infty} \mu(y)+\sum_{x=k^{\prime}+1}^{k-1} x^{a} \mu(x) \sum_{y=k-x}^{\infty} \mu(y) \\
& \leq \sum_{x=1}^{k-1} x \mu(x) \sum_{y=k-x}^{k-1} y^{a-1} \mu(y)+\frac{1}{k} \sum_{x=1}^{k-1} x \mu(x) \sum_{y=k}^{\infty} y^{a} \mu(y)+ \\
& \sum_{x=1}^{k^{\prime}} x^{a} \mu(x) \sum_{y=k-k^{\prime}}^{\infty} \mu(y)+\sum_{x=k^{\prime}+1}^{k-1} x^{a} \mu(x) \sum_{y=1}^{\infty} \mu(y)
\end{aligned}
$$

and

$$
\begin{aligned}
& \sum_{x=1}^{k-1} x \mu(x) \sum_{y=k-x}^{k-1} y^{a-1} \mu(y)=\sum_{y=1}^{k-1} y^{a} \mu(y) \frac{1}{y} \sum_{x=k-y}^{k-1} x \mu(x) \\
& =\sum_{y=1}^{k^{\prime}} y^{a} \mu(y) \frac{1}{y} \sum_{x=k-y}^{k-1} x \mu(x)+\sum_{y=k^{\prime}+1}^{k-1} y^{a} \mu(y) \frac{1}{y} \sum_{x=k-y}^{k-1} x \mu(x) \\
& \leq \sum_{y=1}^{k^{\prime}} y^{a} \mu(y)\left[\sup _{x \geq k-k^{\prime}} x \mu(x)\right]+\sum_{y=k^{\prime}+1}^{k-1} y^{a} \mu(y)\left[\sup _{x} x \mu(x)\right]
\end{aligned}
$$

so that

$$
\begin{gathered}
\frac{1}{C} \sum_{x=1}^{k-1} \mu(x) \sum_{y=k-x}^{\infty} \frac{K(x, y)}{y} \mu(y) \leq \sum_{y=1}^{\infty} y^{a} \mu(y)\left[\sup _{x \geq k-k^{\prime}} x \mu(x)\right]+ \\
\quad \sum_{y=k^{\prime}+1}^{\infty} y^{a} \mu(y)\left[\sup _{x} x \mu(x)\right]+\sum_{y=k}^{\infty} y^{a} \mu(y)\left[\sup _{x} x \mu(x)\right]+ \\
\quad \sum_{x=1}^{\infty} x^{a} \mu(x) \sum_{y=k-k^{\prime}}^{\infty} \mu(y)+\sum_{x=k^{\prime}+1}^{\infty} x^{a} \mu(x) \sum_{y=1}^{\infty} \mu(y)
\end{gathered}
$$

and 4.30 follows.

Corollary 4.13. If 4.29,

$$
\sum_{y=1}^{\infty} y^{a} \mu(y)<\infty
$$


and

$$
\lim _{k \rightarrow \infty} k \mu(k)=0
$$

then

$$
\lim _{k \rightarrow \infty} \sum_{x=1}^{k-1} \mu(x) \sum_{y=k-x}^{\infty} \frac{K(x, y)}{y} \mu(y)=0 .
$$

Proof. Choose $k^{\prime}:=[k / 2]$. Since

$$
\lim _{k \rightarrow \infty} \sum_{y=k^{\prime}}^{\infty} y^{a} \mu(y)=0 \quad \text { and } \quad \lim _{k \rightarrow \infty} \sup _{x \geq k-k^{\prime}} x \mu(x)=0,
$$

the assertion follows from Lemma 4.12 .

Remark 4.14. If $K(x, y) \geq C_{1} x y^{a}$, then

$$
\sum_{x=1}^{k-1} x \mu(x) \sum_{y=k-x}^{k-1} y^{a-1} \mu(y) \geq C_{1}(k-1) \mu(k-1) \mu(1),
$$

so that condition (4.32) is necessary for (4.33). Condition (4.31) with $a=1$ is sufficient for 4.32) (cf. Corollary 4.11).

Since, according to Lemma 4.12 ,

$$
\begin{aligned}
S(k, \gamma, \nu(s))= & \sum_{x=1}^{k-1} \nu(s, x, \gamma) \sum_{y=k-x}^{\infty} \frac{K(x, y, \gamma)}{y} \nu(s, y, \gamma) \leq \\
& C(\gamma) \sum_{x=1}^{\infty} x^{a(\gamma)} \nu(s, x, \gamma)\left[3\left(\sup _{x} x \nu(s, x, \gamma)\right)+2\right],
\end{aligned}
$$

assumptions (3.22), (3.23) and the dominated convergence theorem imply

$$
\lim _{k \rightarrow \infty} \int_{u}^{t} S(k, \gamma, \nu(s)) d s=\int_{u}^{t} \lim _{k \rightarrow \infty} S(k, \gamma, \nu(s)) d s .
$$

On the other hand, 3.22 and 3.23 imply

$$
\sum_{y=1}^{\infty} y^{a(\gamma)} \nu(s, y, \gamma)<\infty \quad \text { and } \quad \lim _{k \rightarrow \infty} k \nu(s, k, \gamma)=0
$$

for almost all $s \in[u, t]$. Thus, the assertion follows from 4.34 and Corollary 4.13 . 


\subsection{Proof of Lemmas 3.5 and 3.7}

Lemma 4.15. The quantities

$$
A_{k}=A_{k}(a, b, \beta)=\sum_{x=1}^{k-1} x^{\beta+a} \sum_{y=k-x}^{\infty} y^{\beta+b-1}, \quad k \geq 2,
$$

where

$$
a, b \in[0,-\beta), \quad \beta<-1,
$$

satisfy

$$
A_{k} \geq-\frac{1}{\beta+b} x_{k}^{\beta+a}\left(k-x_{k}\right)^{\beta+b}(k-1), \quad \forall k \geq 2
$$

and

$$
\begin{aligned}
A_{k} \leq & \frac{\beta+b-1}{\beta+b}\left\{\left(k-x_{k}\right)^{\beta+b}\left[1+\frac{1}{\beta+a+1}\left(x_{k}^{\beta+a+1}-1\right)\right]+\right. \\
& \left.x_{k}^{\beta+a}\left[1+\frac{1}{\beta+b+1}\left(\left(k-x_{k}\right)^{\beta+b+1}-1\right)\right]\right\}, \\
& \forall k \geq 2: \quad 1<x_{k}<k-1,
\end{aligned}
$$

where

$$
x_{k}=\frac{(\beta+a) k}{2 \beta+a+b} .
$$

If $\beta+a+1=0$ or $\beta+b+1=0$, then the undefined terms in (4.38) are to be replaced according to $\lim _{y \rightarrow 0}\left(x^{y}-1\right) / y=\log x$.

Proof. Note that

$$
\sum_{y=k-x}^{\infty} y^{\beta+b-1} \geq \int_{k-x}^{\infty} y^{\beta+b-1} d y=-\frac{1}{\beta+b}(k-x)^{\beta+b}
$$

and

$$
\begin{aligned}
& \sum_{y=k-x}^{\infty} y^{\beta+b-1} \leq(k-x)^{\beta+b-1}+\int_{k-x}^{\infty} y^{\beta+b-1} d y= \\
& \quad(k-x)^{\beta+b-1}-\frac{1}{\beta+b}(k-x)^{\beta+b} \leq \frac{\beta+b-1}{\beta+b}(k-x)^{\beta+b} .
\end{aligned}
$$

Thus, one obtains

$$
A_{k} \geq-\frac{1}{\beta+b} \sum_{x=1}^{k-1} x^{\beta+a}(k-x)^{\beta+b}
$$


and

$$
A_{k} \leq \frac{\beta+b-1}{\beta+b} \sum_{x=1}^{k-1} x^{\beta+a}(k-x)^{\beta+b} .
$$

The function $f(x)=x^{\beta+a}(k-x)^{\beta+b}$ satisfies

$$
f^{\prime}(x)=x^{\beta+a-1}(k-x)^{\beta+b-1}[(\beta+a)(k-x)-x(\beta+b)]
$$

and takes its minimum at $x=x_{k}(\mathrm{cf} .4 .39)$ ). One obtains

$$
\sum_{x=1}^{k-1} x^{\beta+a}(k-x)^{\beta+b} \geq x_{k}^{\beta+a}\left(k-x_{k}\right)^{\beta+b}(k-1)
$$

so that 4.40 implies 4.37).

The function $x^{\beta+a}$ is decreasing and the function $(k-x)^{\beta+b}$ is increasing on $(0, k)$. Thus, one obtains

$$
\begin{aligned}
& \sum_{x=1}^{k-1} x^{\beta+a}(k-x)^{\beta+b}= \\
& \quad \sum_{1 \leq x \leq x_{k}} x^{\beta+a}(k-x)^{\beta+b}+\sum_{x_{k}<x \leq k-1} x^{\beta+a}(k-x)^{\beta+b} \\
& \leq\left(k-x_{k}\right)^{\beta+b} \sum_{1 \leq x \leq x_{k}} x^{\beta+a}+x_{k}^{\beta+a} \sum_{x_{k}<x \leq k-1}(k-x)^{\beta+b} .
\end{aligned}
$$

Note that (if $\beta+a \neq-1$ )

$$
\sum_{1 \leq x \leq x_{k}} x^{\beta+a} \leq 1+\int_{1}^{x_{k}} x^{\beta+a} d x=1+\frac{1}{\beta+a+1}\left(x_{k}^{\beta+a+1}-1\right)
$$

and (if $\beta+a=-1$ )

$$
\sum_{1 \leq x \leq x_{k}} x^{\beta+a} \leq 1+\log x_{k}
$$

In analogy, one obtains (if $\beta+b \neq-1$ )

$$
\begin{aligned}
\sum_{x_{k}<x \leq k-1}(k-x)^{\beta+b} & = \\
\sum_{1 \leq x<k-x_{k}} x^{\beta+b} & \leq 1+\frac{1}{\beta+b+1}\left(\left(k-x_{k}\right)^{\beta+b+1}-1\right)
\end{aligned}
$$

and (if $\beta+b=-1$ )

$$
\sum_{x_{k}<x \leq k-1}(k-x)^{\beta+b} \leq 1+\log \left(k-x_{k}\right) .
$$

Finally, 4.38 follows from 4.41) and 4.42)-4.46). 
Remark 4.16. Note that $1<x_{k}<k-1$ (cf. (4.38)) is fulfilled for sufficiently large $k$, since $(\beta+a) /(2 \beta+a+b) \in(0,1)$, according to 4.36).

Remark 4.17. If $a=b=1$ and $\beta=-3 / 2$, then $x_{k}=k / 2$ (cf. 4.39)) and Lemma 4.15 implies

$$
\frac{4(k-1)}{k} \leq A_{k} \leq 6\left[2-(k / 2)^{-1 / 2}\right]
$$

Corollary 4.18. The expressions 4.35) satisfy

$$
\begin{array}{r}
\lim _{k \rightarrow \infty} A_{k}(a, b, \beta)=0, \quad \text { if } \beta<-\frac{a+b+1}{2}, \\
\liminf _{k \rightarrow \infty} A_{k}(a, b, \beta)>0 \quad \text { and } \quad \limsup _{k \rightarrow \infty} A_{k}(a, b, \beta)<\infty, \\
\text { if } \beta=-\frac{a+b+1}{2},
\end{array}
$$

and

$$
\lim _{k \rightarrow \infty} A_{k}(a, b, \beta)=\infty, \quad \text { if } \beta>-\frac{a+b+1}{2} .
$$

Remark 4.19. The lower and upper bound in (4.47) can be specified in terms of $a, b, \beta$.

One obtains (cf. 4.35)

$$
\begin{aligned}
S(k, \gamma, \nu(s)) & =\sum_{x=1}^{k-1} \nu(s, x, \gamma) \sum_{y=k-x}^{\infty} \frac{K(x, y, \gamma)}{y} \nu(s, y, \gamma) \\
& \leq C \tilde{C}(s)^{2}\left[A_{k}(a, b, \beta)+A_{k}(b, a, \beta)\right]
\end{aligned}
$$

and

$$
S(k, \gamma, \nu(s)) \geq C \tilde{C}(s)^{2}\left[A_{k}(a, b, \beta)+A_{k}(b, a, \beta)\right] .
$$

Now 4.48 implies

$$
\sup _{k \geq l} S(k, \gamma, \nu(s)) \leq C \tilde{C}(s)^{2} \sup _{k \geq l}\left[A_{k}(a, b, \beta)+A_{k}(b, a, \beta)\right]
$$

so that

$$
\lim _{l \rightarrow \infty} \int_{u}^{t} \sup _{k \geq l} S(k, \gamma, \nu(s)) d s=0 \quad \text { if } \quad \beta<-\frac{a+b+1}{2},
$$

according to Corollary 4.18 , and Lemma 3.5 follows. On the other hand, 4.49 implies

$$
\inf _{k \geq l} S(k, \gamma, \nu(s)) \geq C \tilde{C}(s)^{2} \inf _{k \geq l}\left[A_{k}(a, b, \beta)+A_{k}(b, a, \beta)\right]
$$

so that

$$
\lim _{l \rightarrow \infty} \int_{u}^{t} \inf _{k \geq l} S(k, \gamma, \nu(s)) d s>0 \quad \text { if } \quad \beta=-\frac{a+b+1}{2}
$$

and

$$
\lim _{l \rightarrow \infty} \int_{u}^{t} \inf _{k \geq l} S(k, \gamma, \nu(s)) d s=\infty \quad \text { if } \quad \beta>-\frac{a+b+1}{2},
$$

according to Corollary 4.18, and Lemma 3.7 follows. 


\section{References}

[1] Aldous, D. (1998). Tree-valued Markov chains and Poisson-Galton-Watson distributions. In Microsurveys in discrete probability (Princeton, NJ, 1997). DIMACS Ser. Discrete Math. Theoret. Comput. Sci., Vol. 41. Amer. Math. Soc., Providence, RI, 1-20. MR1630406

[2] Aldous, D. J. (1999). Deterministic and stochastic models for coalescence (aggregation and coagulation): a review of the mean-field theory for probabilists. Bernoulli 5, 1, 3-48. MR1673235

[3] Deaconu, M. and Fournier, N. (2002). Probabilistic approach of some discrete and continuous coagulation equations with diffusion. Stochastic Process. Appl. 101, 1, 83-111. MR1921443

[4] Eibeck, A. ANd Wagner, W. (2001). Stochastic particle approximations for Smoluchowski's coagulation equation. Ann. Appl. Probab. 11, 4, 1137-1165. MR1878293

[5] Eibeck, A. And Wagner, W. (2003). Stochastic interacting particle systems and nonlinear kinetic equations. Ann. Appl. Probab. 13, 3, 845-889. MR1994039

[6] Ernst, M. H., Ziff, R. M., And Hendriks, E. M. (1984). Coagulation processes with a phase transition. J. Colloid Interface Sci. 97, 1, 266-277.

[7] Escobedo, M., Laurençot, P., Mischler, S., and Perthame, B. (2003). Gelation and mass conservation in coagulation-fragmentation models. J. Differential Equations 195, 1, 143-174. MR2019246

[8] Escobedo, M., Mischler, S., And Perthame, B. (2002). Gelation in coagulation and fragmentation models. Comm. Math. Phys. 231, 1, 157-188. MR1947695

[9] Ethier, S. N. and Kurtz, T. G. (1986). Markov Processes, Characterization and Convergence. Wiley, New York. MR838085

[10] Flory, P. J. (1941). Molecular size distribution in three dimensional polymers. I. Gelation. J. Am. Chem. Soc. 63, 3083-3090.

[11] Flory, P. J. (1946). Fundamental principles of condensation polymerization. Chem. Revs. 39, 137-197.

[12] Flory, P. J. (1953). Principles of polymer chemistry. Cornell University Press, New York.

[13] Fournier, N. And Giet, J.-S. (2004). Convergence of the Marcus-Lushnikov process. Methodol. Comput. Appl. Probab. 6, 2, 219-231. MR2035293

[14] Gillespie, D. T. (1972). The stochastic coalescence model for cloud droplet growth. J. Atmospheric Sci. 29, 1496-1510.

[15] Guiaş, F. (2001). Convergence properties of a stochastic model for coagulationfragmentation processes with diffusion. Stochastic Anal. Appl. 19, 2, 245-278. MR1841439

[16] Hendriks, E. M., Ernst, M. H., And Ziff, R. M. (1983). Coagulation equations with gelation. J. Statist. Phys. 31, 3, 519-563. MR711489 
[17] Herrero, M. A., Velázquez, J. J. L., and Wrzosek, D. (2000). Sol-gel transition in a coagulation-diffusion model. Phys. D 141, 3-4, 221-247. MR1760997.

[18] Lang, R. And NGuyen, X. X. (1980). Smoluchowski's theory of coagulation in colloids holds rigorously in the Boltzmann-Grad limit. Z. Wahrsch. Verw. Gebiete 54, 227-280. MR602510

[19] Laurençot, P. And Mischler, S. (2002). The continuous coagulation-fragmentation equations with diffusion. Arch. Ration. Mech. Anal. 162, 1, 45-99. MR1892231

[20] Laurençot, P. and Wrzosek, D. (2002). From the nonlocal to the local discrete diffusive coagulation equations. Math. Models Methods Appl. Sci. 12, 7, 1035-1048. MR1918172

[21] Lushnikov, A. A. (1978). Certain new aspects of the coagulation theory. Izv. Akad. Nauk SSSR Ser. Fiz. Atmosfer. i Okeana 14, 10, 738-743.

[22] Marcus, A. H. (1968). Stochastic coalescence. Technometrics 10, 1, 133-143. MR223151

[23] Norris, J. R. (2000). Cluster coagulation. Comm. Math. Phys. 209, 2, 407-435. MR1737990

[24] Norris, J. R. (2004). Brownian coagulation. Commun. Math. Sci. 2, suppl. 1, 93-101. MR2119877

[25] Siegmund-Schultze, R. And Wagner, W. (2006). Induced gelation in a two-site spatial coagulation model. Ann. Appl. Probab. 16, 1, 370-402. MR2209346

[26] Stockmayer, W. H. (1943). Theory of molecular size distribution and gel formation in branched-chain polymers. J. Chem. Phys. 11, 2, 45-55.

[27] van Dongen, P. G. J. (1989). Spatial fluctuations in reaction-limited aggregation. J. Statist. Phys. 54, 1-2, 221-271. MR984257

[28] van Dongen, P. G. J. And Ernst, M. H. (1986). On the occurrence of a gelation transition in Smoluchowski's coagulation equation. J. Statist. Phys. 44, 5-6, 785-792. MR858257

[29] van Dongen, P. G. J. And Ernst, M. H. (1987). Fluctuations in coagulating systems. J. Statist. Phys. 49, 5-6, 879-926. MR935480

[30] von Smoluchowski, M. (1916). Drei Vorträge über Diffusion, Brownsche Molekularbewegung und Koagulation von Kolloidteilchen. Phys. Z. 17, 557-571, 585-599.

[31] Wagner, W. (2005). Explosion phenomena in stochastic coagulation-fragmentation models. Ann. Appl. Probab. 15, 3, 2081-2112. MR2152254

[32] ZIFF, R. M. (1980). Kinetics of polymerization. J. Statist. Phys. 23, 2, 241-263. MR586509

[33] Ziff, R. M., Hendriks, E. M., And Ernst, M. H. (1982). Critical properties for gelation: A kinetic approach. Phys. Rev. Lett. 49, 8, 593-595.

[34] Ziff, R. M. And Stell, G. (1980). Kinetics of polymer gelation. J. Chem. Phys. 73, 7, 3492-3499. 